\title{
EXTENDED CELL-TRANSMISSION-BASED EVACUATION PLANNING IN URBAN AREAS*
}

\author{
Alf Kimms** and Klaus-Christian Maassen
}

Received September 1, 2011 / Accepted October 3, 2011

\begin{abstract}
A well-known traffic simulation model bases on the idea to represent street sections by socalled cells where vehicles are streaming from one cell to another in order to give a realistic image of traffic flows. Its basic ideas were picked up recently to formulate an optimization model for evacuation planning purposes. We will address three major weaknesses of the recent model, namely the usage of a fixed single cell size, missing consideration of lanes as well as missing limitations for traffic flows and show how to overcome them. Stimulated by this, we formulate the extended Cell-Transmission-Based Evacuation Planning Model and a new optimization model to facilitate this model in terms of choosing the optimum cell size. Additionally, we will present an iterative simulation-based optimization approach in order to evaluate and to enhance results in a more realistic environment. This approach takes advantage of a standalone vehicle reallocation optimization model, additional traffic flow limitations as well as a state-of-the-art microscopic traffic simulation. We will demonstrate the effectiveness of this approach in a real-world example.
\end{abstract}

Keywords: evacuation, urban area, cell-transmission-model, iterative heuristic, optimization, traffic simulation.

\section{INTRODUCTION}

In the last few decades risk potential for societies permanently increased due to threats like extreme weather conditions, natural disasters, terror attacks or chemical accidents. Evacuations gain more and more interest to ensure protection for people concerned. But, this protective function only can be provided if several aspects are considered. In particular, it has to be determined which area must be evacuated, how many exits are available, how to allocate evacuees to these exits and how to route traffic. Decision-making in such problems is very challenging due to almost unpredictable behavior of evacuees, complex traffic dynamics as well as the basic conditions of the evacuation (e.g. time of day, season).

\footnotetext{
*Invited paper

**Corresponding author

Lehrstuhl für Betriebswirtschaftslehre, insbsondere Logistik und Operations Research, Mercator School of Management, University of Duisburg-Essen, Lotharstr. 65, 47048 Duisburg, Germany.

E-mails: alf.kimms@uni-due.de / klaus-christian.maassen@uni-due.de
} 
In the past, there were numerous publications concerning evacuation problems in general (see [8] and [12] for reviews of evacuation from buildings and ships) but only a small fraction aimed at the evacuation of urban areas. Further differentiation between traffic simulation models and analytical approaches can be made here.

In the range of traffic simulation models, Sheffi et al. [22] were one of the first to simulate the evacuation of large areas using basic traffic dynamics. Later, Sinuany-Stern \& Stern [24] simulated the evacuation of a small city with 33 junctions and 51 street sections considering both vehicles and pedestrians. Although not investigating evacuation in particular, the cellular automata (CA) Model by Nagel \& Schreckenberg [19] was the first simulation model to describe "traffic jams appearing from nowhere" and is still used to simulate vehicle and pedestrian movements (e.g. [2]). At this point, we want to clear that our approach also uses "cells" like CA, but the fundamental ideas behind this representation are totally different. Although Nagel \& Rickert [18] proved large scale applicability of CA, it has major disadvantages in terms of optimization purposes due to enormous computational effort caused by the very high number of required cells. The common weakness of all models mentioned above and all traffic simulation models in general is that (due to the nature of a simulation) simulation approaches alone can not be utilized for optimization purposes. Traffic simulation models are perfectly suited for detailed and realistic illustration and evaluation but not for optimization aims, which are required here.

Yamada [28] proposed two simple network flow methods for the evacuation of a big japanese city which does not consider traffic dynamics at all. Cova \& Johnson [5] introduced a lane-based network flow model for evacuation planning. So far, evacuees were assigned to their closest exits in order to minimize the total travel distance. However, Han et al. [9] showed that such assignments can be suboptimal, if e.g. roads are congested due to traffic dynamics. Andreas \& Smith [1] used a network tree approach under consideration of multiple scenarios to generate evacuation routes. Stepanov \& MacGregor Smith [25] combined an IP Model for route assignment with $M / G / c / c$ queuing models to capture congestion and time delays during an evacuation. Sbayti \& Mahmassani investigated the benefit of scheduled evacuation planning ([21]). MurrayTuite \& Mahmassani study the impact of human behavior, e.g. the intention to drive home to secure valuables or to consolidate with other family members before evacuating, see [16] and [17]. However, suchlike aspects will be neglected in this paper.

A popular approach for evacuation planning and traffic assignment in general is the Cell-Transmission-Model by Daganzo ([6,7]) which bases on the hydrodynamic traffic flow model by Lighthill, Whitham \& Richards $([13,20])$. The basic ideas are as follows: a given road network is segmented into cells whereas the cell length is chosen to be a distance which can be passed by a vehicle in one period under free flow conditions. Furthermore, a maximum capacity as well as a maximum in- and outflow per period is assigned to each cell. Now, traffic flows under consideration of traffic dynamics like congestion, merging and diverging effects - are created by vehicles moving from cell to cell. Thus, the optimization models on the basis of the CTM approach can be interpreted as an optimizable macroscopic traffic illustration. There are numerous publications utilizing the basic ideas of the CTM, e.g. $[4,10,15,23,26,27,31]$ : Chiu 
et al. [4], Kalafatas \& Peeta [10], Liu et al. [15], Shen et al. [23], Tuydes \& Ziliaskopoulos [26] and Xie et al. [27] presented optimization models based on the Cell-Transmission equations for evacuation planning which were applied to networks of different size. The seminal work of Ziliaskopoulos [31] contains a general model for dynamic traffic assignment problems.

However, the Cell-Transmission-based optimization model for evacuation planning in this paper is different from the previously mentioned models in many ways. Firstly, we use a more flexible objective function that covers different danger levels $c_{i t}$ for a cell $i$ in period $t$ in the evacuation zone. Secondly, the model is formulated without defining a separate set of so-called connectors (for merging, diverging, ordinary, source and sink cells). Instead, we are using a single binary parameter that denotes whether a physical connection between two cells exists. Thirdly, we do not predetermine predecessors and successors of a cell, because this is part of the optimization process. This is the most relevant difference between our model formulation and the previous work, since we assume that the evacuation traffic routing is not known in advance. ([27] defined the evacuation routing in the upper level of their bi-level approach.) Partially, lane reversals are taken into account, also affecting traffic routing, see e.g. [10] or [26]. Fourthly, in contrast to the papers discussed above, we integrate the departure times as an own decision variable into the model. Fifthly, we add three new enhancements, namely multiple cell sizes, explicit consideration of lanes as well as limitations for merging and diverging traffic flows to improve real world applicability. Sixthly, we introduce a new optimization model to determine the optimum cell size for a given network. At this point, we want to emphasize that we pick up the basic ideas of Daganzo's Cell-Transmission approach, but we do not claim that our optimization model (with multiple cell sizes and limited traffic routing options) shares all characteristic properties of the original CTM by Daganzo. In contrast to all already mentioned papers where traffic routing decisions are already done, our aim is to develop a framework to optimize and supplement traffic routing decisions in the case of real-world evacuation scenarios.

The paper is structured as follows: In Section 2 we introduce two approaches to enhance the Cell-Transmission-based Evacuation Planning Model (CTEPM) by Kimms \& Maassen [11] and formulate the Extended Cell-Transmission-based Evacuation Planning Model (ExCTEPM) by integrating these approaches. This section also contains a simple optimization model to compute optimum cell sizes for a given network as well as a numerical example. In Section 3, we utilize a simulation-based optimization approach which combines the ExCTEPM, a vehicle reallocation optimization model, additional traffic flow limitations and SUMO as a state-of-theart open source microscopic traffic simulation (see http://sumo.sourceforge.net) in an iterative procedure. Afterwards, the computational study in Section 4 demonstrates the benefits of our integrated simulation-based optimization approach. Finally, Section 5 includes some summarizing conclusions.

\section{EXTENDING THE CTEPM}

The CTEPM has two major weaknesses. On the one hand, the use of cells with a fixed single size may lead to several disadvantages in terms of an unnecessary high number of (relatively 
small) cells to meet a predetermined accuracy level in network representation and to excessive amounts of constraints and variables in the optimization model caused by the very high number of required cells, respectively. On the other hand, missing consideration of lanes, limitations for merging and diverging traffic flows as well as traffic flow redirections during the planning horizon may result in solutions that may be optimal regarding the optimization model, but may also be inappropriate or even infeasible in real-world applications. We will discuss these issues in detail in Section 2.2. In the following text we will first show how to implement multiple cell sizes into the model and demonstrate the benefits of this approach. Subsequently, explicit consideration of lanes as well as traffic flow limitations will be discussed and implemented to formulate the EXCTEPM.

\subsection{Multiple Cell Sizes}

In order to represent a given network with the CTEPM, it is necessary to choose a cell size which matches the length of the network arcs adequately. As can easily imagined, this will lead to a tradeoff between the number of cells (which immensely affects the number of side constraints and variables) and the accuracy of the network representation. In this paper, we will extend the CTEPM by the usage of multiple cell sizes whereas larger cell sizes will be integer multiples of the reference (standard) cell size due to the discrete nature of the Cell-Transmission approach.

Figures 1 and 2 illustrate this idea in the case of a very small network. There, we model four street sections at $50 \mathrm{~km} / \mathrm{h}$ and $30 \mathrm{~km} / \mathrm{h}$ driving speed, respectively. As can be seen easily, the number of required cells can be lowered from eight to five cells. In this example, vehicles need two periods to pass large cells, of course.

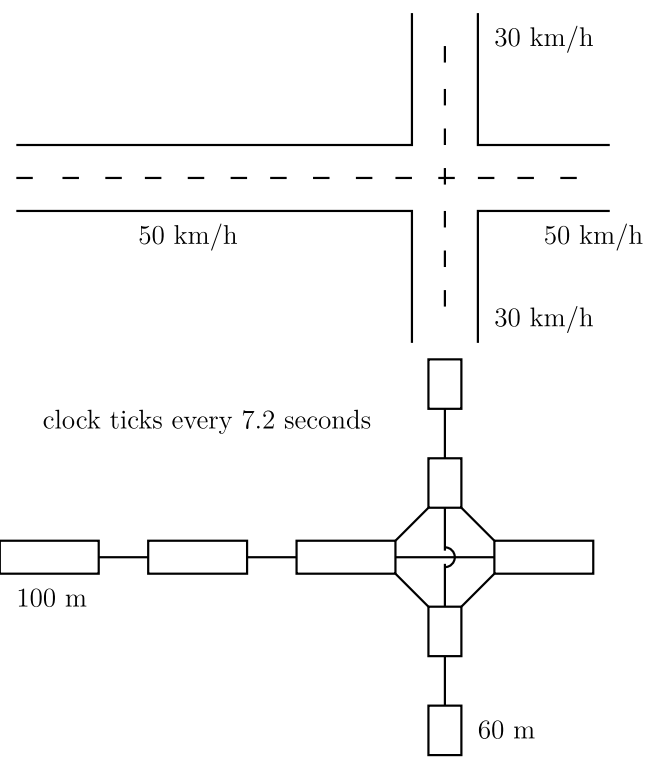

Figure 1 - Network Representation Using One Cell Size. 


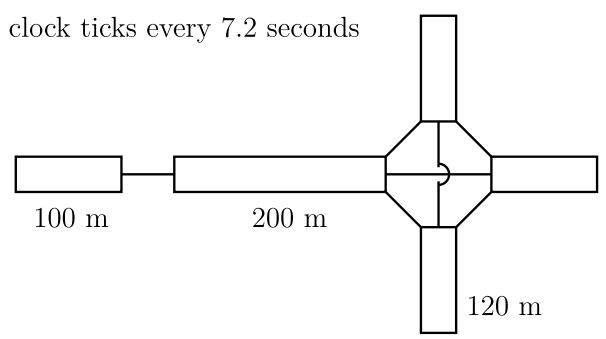

Figure 2 - Network Representation Using Two Cell Sizes.

In the mathematical formulation, we assume to use $|N|$ different cell sizes, where a certain cell size $n$ is $n$-times larger than cell size 1. For example, $|N|=3$ leads to three different cell sizes (and three set of cells, namely $\left.I_{1}, I_{2}, I_{3}\right)$, where all cells $i \in I_{2}\left(I_{3}\right)$ will be two-times (threetimes) larger than cells $i \in I_{1}$, if we assume the same driving speed. As can be seen in Figure 1 and 2 several cells can be combined to a larger cell without any loss in network representation accuracy.

This approach results in the following advantages: firstly, the accuracy of the network representation using the same number of cells can be improved since a smaller standard cell size (and integer multiples of this cell size) tends to match the underlying arc lengths more accurate, if the same number of cells is used as before. Moreover, the same accuracy in network representation can be achieved with less cells since standard sized cells can be merged into one larger cell. Secondly, the computational effort can be reduced due to a usually smaller number of required cells. Admittedly, the mathematical formulation of the (extended) CTEPM will get more complex, but the advantages of the reduced number of cells still prevail as can be seen later in this section and in Section 2.4.

We will first present the CTEPM as written in Kimms \& Maassen [11]. Afterwards, we will introduce two different approaches to capture multiple cell sizes in the CTEPM.

Parameters:

$\begin{array}{ll}T=\{1, \ldots,|T|\} & \begin{array}{l}\text { index set of periods (where }|T| \text { is the index of the last period } \\ \text { of the planning horizon) }\end{array} \\ N=\{1, \ldots,|N|\} & \begin{array}{l}\text { index set of different cell size multipliers (where }|N| \text { denotes } \\ \text { the maximum cell size) }\end{array} \\ I=\{1, \ldots,|I|\} & \begin{array}{l}\text { index set of cells } \\ \text { index set of cells of cell size } n\left(\bigcup_{n \in N} I_{n}=I\right)\end{array} \\ I_{n} & \begin{array}{l}\text { index of super sink } \\ |I|\end{array} \\ I_{S} & \begin{array}{l}\text { index set of sink cells }\left(I_{S}=\left\{i \in I: \beta_{i,|I|}=1\right\}\right) \\ c_{i t}\end{array} \\ \beta_{i j} & =\beta_{j i}=1, \text { if a traffic flow from cell } i \text { to cell } j \\ & \text { (and vice versa) can be established }(0, \text { otherwise) } \\ N_{i t} & \text { maximum vehicle capacity of cell } i \text { (on all lanes) in period } t\end{array}$


$E_{i} \quad$ number of vehicles starting their evacuation in cell $i$ (derived from population in cell $i$ )

$Q_{i t}$ maximum number of in- and outflowing vehicles (on all lanes) per period into/from cell $i$ in period $t$

$x_{i 1} \quad$ number of vehicles en route at cell $i$ at the end of period 1, i.e. before the evacuation starts

Decision Variables:

$z_{i t} \quad$ number of vehicles residing, leaving or waiting in cell $i$ in period $t$

$x_{i t} \quad$ number of residing vehicles at cell $i$ at the end of period $t$

$y_{i j t} \quad$ number of vehicles leaving section $i$ in period $t$ and reaching section $j$ in period $t+1$

$b_{i t} \quad$ number of vehicles starting their evacuation at cell $i$ in period $t$

Given this notation multiple cell sizes can be implemented in the CTEPM as follows:

$$
\min \sum_{i \in I} \sum_{t \in T} z_{i t} \cdot c_{i t}
$$

subject to

$$
\begin{aligned}
& z_{i t}=x_{i t}+\sum_{j \in I} y_{i j t}+\left(E_{i}-\sum_{\tau=1}^{t} b_{i \tau}\right) \quad i \in I ; t \in T \\
& x_{i t}=b_{i t}+x_{i, t-1}+\sum_{j \in I} y_{j i, t-1}-\sum_{j \in I} y_{i j t} \quad i \in I ; t=2, \ldots,|T| \\
& z_{i t} \leq N_{i t}+\left(E_{i}-\sum_{\tau=1}^{t} b_{i \tau}\right) \quad i \in I ; t \in T \\
& x_{i t} \leq N_{i t} \quad i \in I ; t \in T \\
& \sum_{t \in T} b_{i t}=E_{i} \quad i \in I \\
& x_{|I|,|T|}=\sum_{i \in I}\left(E_{i}+x_{i 1}\right) \\
& \sum_{i \in I} y_{i j t} \leq\left(N_{j t}-x_{j t}\right) \quad j \in I ; t \in T \\
& \sum_{i \in I} y_{i j t} \leq Q_{j t} \quad j \in I ; t \in T \\
& \sum_{j \in I} y_{i j t} \leq Q_{i t} \quad i \in I ; t \in T \\
& y_{i j t} \leq N_{i t} \cdot \beta_{i j} \quad i, j \in I ; t \in T
\end{aligned}
$$




$$
\begin{array}{ll}
z_{i t}, x_{i t}, b_{i t} \geq 0 & i \in I ; t \in T \\
y_{i j t} \geq 0 & i, j \in I ; t \in T \\
x_{i 1}=0 & i \in I \\
b_{i 1}=0 & i \in I \\
y_{i j 1}=0 & i \in I ; j \in I
\end{array}
$$

The objective function (1) minimizes total danger for all vehicles during the complete planning horizon. Here, the parameter $c_{i t}$ might be related to some key figures, e.g. the probability that an area will be reached by a tsunami wave or the concentration of hazardous material at a certain point of the planning horizon, see [3] and [30]. The case of $c_{i t}=c_{i} \cdot t$ may be used for scenarios with no movement of danger during the planning horizon, e.g. a preventive evacuation. However, using general values for $c_{i t}$ is more flexible since it allows to capture movement of danger during in the planning horizon, e.g. movement of a hazardous plume over time. The calculation of all vehicles residing, leaving or waiting in cell $i$ is done in equation (2). Vehicleflow-constraints have to be defined with respect to the cell size $n$. For $n=1$, the standard vehicle flow constraint from the single cell size CTEPM can be adopted (see (3)), whereas the number of residing vehicles at the end of period $t$ equals the number of vehicles starting their evacuation in period $t$ plus the number of residing vehicles in cell $i$ at the end of period $t-1$ plus the number of inflowing vehicles reaching cell $i$ in period $t$ minus the number of outflowing vehicles leaving cell $i$ till the end of period $t$, respectively. This vehicle flow equation also allows vehicle holding, if this would be beneficial with regards to the objective value.

Moreover, (4) ensures that the number of vehicles (incl. all vehicles that have not left their homes, yet) in a cell $i$ during a period $t$ does not exceed the maximum capacity of this cell plus the number of vehicles which have not entered the network so far. Similar to (4), constraint (5) forces the number of vehicles in cell $i$ at the end of period $t$ to be lower than the maximum capacity of this cell. (6) determines the departure schedule for each cell and that all residents will leave their homes. Furthermore, equation (7) assures that each vehicle (residents in vehicles in terms of $E_{i}$ and initial traffic (e.g. due to rush hour traffic) in terms of $x_{i 1}$ ) will be evacuated. Here, it is important that $|T|$ is sufficiently large so that all vehicles can escape within the planning horizon. In (8), the maximum number of cell-changing vehicles is limited to the remaining capacity (= maximum vehicle capacity minus the number of vehicles at the end of a period) of the corresponding cells. As stated in (9) and (10) the maximum number of in- and outflowing vehicles additionally must be limited by the in- and outflow capabilities of a cell. In the following, we assume that flow capacities are constant over time so that using $Q_{i}$ (instead of $Q_{i t}$ ) is sufficient. Furthermore, traffic flows can stream from a cell $i$ to cell $j$, if and only if, a physical connection between these cells exist, i.e. these cells are adjacent - see (11). (12) and (13) finally determine the domains of the decision variables. Please note that it is sufficient to consider only those flow variables $y_{i j t}$ where $\beta_{i j}=1$ holds. 
In order to assure that no vehicle movements occur in the first period - where the evacuation has not started yet - , boundary conditions (14)-(16) fix the decision variables $x_{i t}, b_{i t}$ and $y_{i j t}$ to zero in the first period. However, if initial traffic is existent, (14) has to be specified for all affected cells.

Although $z_{i t}, x_{i t}, b_{i t}$ and $y_{i j t}$ can be defined to take only integer values, we decided to relax integrality. Firstly, this relaxation helps to reduce computational effort due to less integer variables. Secondly, in all computational tests throughout this article - all parameters of the ExCTEPM are set to integer values - all variables which will be used in the succeeding simulation runs take only integer values automatically, so that there was no need to restore integrality constraints of the decision variables. In detail, three variables are passed to the simulation. First, let $\eta_{i j}$ denote the total number of vehicles traveling from cell $i$ to cell $j$ during the complete planning horizon, i.e. $\eta_{i j}=\sum_{t \in T} y_{i j t}$. Secondly, the driving direction is expressed by $\varphi_{i j}\left(\varphi_{i j} \in\{0,1\}\right)$ indicating if a connection from cell $i$ to cell $j$ is used $\left(\varphi_{i j}=1\right)$. Thirdly, assigned lanes between cell $i$ and cell $j$ are denoted by $\epsilon_{i j}\left(\epsilon_{i j} \in \mathbb{N}_{0}\right)$. The variables $z_{i t}, x_{i t}, b_{i t}$ from the optimization part will be ignored in the simulation. Since $\varphi_{i j}$ and $\epsilon_{i j}$ are set to integer values by definition, only $\eta_{i j}$ may take non-integer values. In such cases, we developed a simple postprocessing approach (see Appendix A) which uses the (integer and/or non-integer) $\eta_{i j}$ values from the optimized solution as a starting point to construct a slightly modified (feasible) solution only consisting of integer values for $\eta_{i j}$.

In order to obtain feasible solutions, $|T|$ must be chosen sufficiently large so that all evacuees have the ability to escape within the planning horizon. A lower bound for $|T|$ can be computed by $|T|_{L B}=\sum_{i \in I} E_{i} / \sum_{i \in I_{S}} Q_{i}$. However, for our computational tests we set $|T|=1.5 \cdot|T|_{L B}$. However, larger values for $|T|$ will not lead to any computational issues.

\subsubsection{Multiple Cell Sizes - Approach 1}

The basic idea of this approach is to divide a cell into subcells, whereas the number of subcells corresponds to the cell size of the cell $(1, \ldots, n)$. A vehicle needs at least one period to pass a subcell so that $n$ subcells lead to a minimum travel time of $n$ periods. In order to capture vehicle movement from subcell to subcell, a new decision variable $y_{i i t}^{k}$ must be introduced. This variable is an auxiliary flow variable which displays the number of vehicles moving from subcell $k$ to subcell $k+1$ in cell $i$ in period $t$. Thus, there must be $n-1$ additional flow variables for a cell of cell size $n$. Now, the CTEPM with multiple cell sizes using this approach can be formulated by replacing equation (2) by (17), (3) by (18) and adding three more constraints (19)-(21):

$$
\begin{array}{ll}
z_{i t}=x_{i t}+\sum_{j \in I} y_{i j t}+\sum_{n \in N} y_{i i t}^{n}+\left(E_{i}-\sum_{\tau=1}^{t} b_{i \tau}\right) & i \in I ; t \in T \\
x_{i t}=b_{i t}+x_{i, t-1}+\sum_{j \in I} y_{j i, t-1}-\sum_{j \in I} y_{i j t} & i \in I_{1} ; t=2, \ldots,|T|
\end{array}
$$




$$
\begin{array}{ll}
x_{i t}=b_{i t}+x_{i, t-1}+\sum_{j \in I} y_{j i, t-1} & \\
-\sum_{k=1}^{n-1} y_{i i t}^{k}+\sum_{k=1}^{n-1} y_{i i, t-1}^{k}-\sum_{j \in I} y_{i j t} & n \in N: n \geq 2 ; i \in I_{n} ; \\
& t=2, \ldots,|T| \\
y_{i i, t-1}^{k}=y_{i i t}^{k+1} & n \in N: n \geq 3 ; k=1, \ldots, n-2 ; \\
& i \in I_{n} ; t=2, \ldots,|T| \\
y_{i i, t-1}^{n-1}=\sum_{j \in I} y_{i j t} & n \in N: n \geq 2 ; i \in I_{n} ; \\
& t=2, \ldots,|T| \\
y_{i i t}^{n} \geq 0 & n \in N ; i \in I ; t \in T
\end{array}
$$

Equation (2) must by replaced by (17) in order to cover the vehicle movements from subcell to subcell in the objective value. The standard vehicle flow equation (3) now only holds for cells of size 1, see (18). For larger cells, an extended vehicle flow equation (19) must be introduced. Vehicle flows from subcell to subcell are captured by (20) and (21). However, this approach has some disadvantages in terms of traffic flow representation. First, traffic holding is only possible in the first subcell, because of (20) and (21) forcing traffic to flow from one subcell to the next subcell in successive periods. Secondly, all evacuees starting their evacuation in a cell of cell size $|N| \geq 2$ also have to pass all subcells before they can leave the cell.

\subsubsection{Multiple Cell Sizes - Approach 2}

The concept of the second approach to capture multiple cell sizes is to limit traffic outflow with respect to traffic inflow of a cell so that a minimum travel time of $n$ periods for a cell of size $n$ can be ensured. Because of (3), this assumption automatically holds for cells with size 1 . For cells of size $n \geq 2$, we introduce constraint (23) to ensure a minimum travel time of $n_{i}$ periods for a cell $i$ of size $n_{i}$. The CTEPM with multiple cell sizes using the seconds approach can be formulated by adding the following constraint to the model formulation (1)-(16):

$$
\begin{gathered}
\sum_{j \in I} \sum_{\tau=1}^{t} y_{i j \tau} \leq \sum_{j \in I} \sum_{\tau=1}^{\max \left(t-n_{i}, 1\right)} y_{j i \tau}+\sum_{\tau=1}^{\max \left(t-\left\lceil n_{i} / 2\right\rceil+1,1\right)} b_{i \tau}+x_{i 1} \\
i \in I: n_{i} \geq 2 ; t=2, \ldots,|T|
\end{gathered}
$$

This constraint limits the cumulated number of vehicles leaving a cell $i$ between period 1 and period $t$ to the cumulated number of vehicles entering the cell between period 1 and period $t-n_{i}$ plus the vehicles starting their evacuation between period 1 and period $t-\left\lceil n_{i} / 2\right\rceil+1$ plus the 
initial street occupation in terms of $x_{i 1}$, where $n_{i}$ is the size of cell $i$. This approach has several advantages compared to the first approach. First, no new variables have to be introduced and less constraints are needed when cells of size $|N| \geq 3$ are used. Secondly, waiting vehicles can escape from a cell immediately after the minimum travel time has passed. Thirdly, evacuees start their evacuation in the "center" of a cell if the term $t-\left\lceil n_{i} / 2\right\rceil+1$ is used. However, this approach allows to modify the "starting point" as desired by the decision maker.

\subsubsection{Impact of Multiple Cell Sizes on Problem Size}

To illustrate the effectiveness of both approaches, we calculate the optimum number of cells for $|N| \in\{1,2,3,4,5,10\}$ in the case of three randomly generated networks of different size applying the cell size optimization model (37)-(47) that will be described in detail in Section 2.3. On the basis of this number we also compute the number of constraints and variables that would emerge in model (1)-(11) plus the corresponding additional constraints to cover multiple cell sizes. The networks consist of 50, 100 and 150 street sections representing a total network length of $15.852 \mathrm{~m}, 30.978 \mathrm{~m}$ and $47.691 \mathrm{~m}$, respectively. In addition, we assume $70 \%(30 \%)$ of the street sections allow a driving speed of $50 \mathrm{~km} / \mathrm{h}(30 \mathrm{~km} / \mathrm{h})$ and that the length of $50 \mathrm{~km} / \mathrm{h}$ $(30 \mathrm{~km} / \mathrm{h})$ sections can take all integer values within the interval $[100 \mathrm{~m}, 700 \mathrm{~m}]([50 \mathrm{~m}, 200 \mathrm{~m}])$. Furthermore, we force the absolute deviation between the street sections lengths and the lengths of the corresponding cells to be lower than $15 \%$ of the street sections length.

In the following, the number of constraints as well as the number of variables are calculated assuming a planning horizon of 600 seconds. The results for the first approach are shown in Tables 1-3 and for the second approach in Tables 4-6. Bracketed percentages display the relative reduction in comparison to the application of only one cell size $(|N|=1)$, which equates the standard CTEPM defined in [11].

Table 1 - Size of Model Formulation (1), (4)-(11), (17)-(21) for a 50-Street-Network.

\begin{tabular}{|c|c|c|c|c|}
\hline \#Cell Sizes & \#Cells & Period Length & \#Constraints & \#Variables \\
\hline$|N|=1$ & 283 & $4.0 \mathrm{sec}$. & $12,310,501$ & $12,140,700$ \\
\hline$|N|=2$ & $153(-45.9 \%)$ & $4.0 \mathrm{sec}$. & $3,692,861(-70.0 \%)$ & $3,601,200(-70.3 \%)$ \\
\hline$|N|=3$ & $107(-62.2 \%)$ & $4.0 \mathrm{sec}$. & $1,865,461(-84.8 \%)$ & $1,793,100(-85.2 \%)$ \\
\hline$|N|=4$ & $94(-66.8 \%)$ & $4.0 \mathrm{sec}$. & $1,479,380(-88.0 \%)$ & $1,398,150(-88.5 \%)$ \\
\hline$|N|=5$ & $76(-73.1 \%)$ & $4.0 \mathrm{sec}$. & $1,010,867(-91.8 \%)$ & $933,300(-92.3 \%)$ \\
\hline$|N|=10$ & $53(-81.3 \%)$ & $4.0 \mathrm{sec}$. & $648,500(-94.7 \%)$ & $481,650(-96.0 \%)$ \\
\hline
\end{tabular}

Two major findings can be derived from these tables: firstly, massive improvements in terms of problem size reduction can be achieved, whereas the additional benefit decreases with increasing values for $|N|$. Secondly, relative benefit is almost the same for all networks and cell sizes $|N|$. Thirdly, relative reduction in problem size is much larger than the relative reduction of cells in all cases.

The results for the second approach shows great similarity to the first one. However, for $|N| \geq 3$ the number of constraints and variables is always lower. 
Table 2 - Size of Model Formulation (1), (4)-(11), (17)-(21) for a 100-Street-Network.

\begin{tabular}{|c|c|c|c|c|}
\hline \#Cell Sizes & \#Cells & Period Length & \#Constraints & \#Variables \\
\hline$|N|=1$ & 798 & $2.7 \mathrm{sec}$. & $143,252,971$ & $142,541,154$ \\
\hline$|N|=2$ & $425(-46.7 \%)$ & $2.7 \mathrm{sec}$. & $41,032,045(-71.4 \%)$ & $40,653,346(-71.5 \%)$ \\
\hline$|N|=3$ & $292(-63.4 \%)$ & $2.7 \mathrm{sec}$. & $19,602,885(-86.3 \%)$ & $19,330,532(-86.4 \%)$ \\
\hline$|N|=4$ & $233(-70.8 \%)$ & $2.7 \mathrm{sec}$. & $12,649,759(-91.2 \%)$ & $12,400,138(-91.3 \%)$ \\
\hline$|N|=5$ & $197(-75.3 \%)$ & $2.7 \mathrm{sec}$. & $9,193,249(-93.6 \%)$ & $8,934,272(-93.7 \%)$ \\
\hline$|N|=10$ & $131(-83.6 \%)$ & $2.7 \mathrm{sec}$. & $4,696,729(-96.7 \%)$ & $4,082,461(-97.1 \%)$ \\
\hline
\end{tabular}

Table 3 - Size of Model Formulation (1), (4)-(11), (17)-(21) for a 150-Street-Network.

\begin{tabular}{|c|c|c|c|c|}
\hline \#Cell Sizes & \#Cells & Period Length & \#Constraints & \#Variables \\
\hline$|N|=1$ & 1,432 & $2.3 \mathrm{sec}$. & $537,829,129$ & $536,334,120$ \\
\hline$|N|=2$ & $758(-47.1 \%)$ & $2.3 \mathrm{sec}$. & $151,537,691(-71.8 \%)$ & $150,747,075(-71.9 \%)$ \\
\hline$|N|=3$ & $528(-63.1 \%)$ & $2.3 \mathrm{sec}$. & $74,009,381(-86.2 \%)$ & $73,435,743(-86.3 \%)$ \\
\hline$|N|=4$ & $416(-70.9 \%)$ & $2.3 \mathrm{sec}$. & $46,293,469(-91.4 \%)$ & $45,789,840(-91.5 \%)$ \\
\hline$|N|=5$ & $345(-75.9 \%)$ & $2.3 \mathrm{sec}$. & $32,133,161(-94.0 \%)$ & $31,656,168(-94.1 \%)$ \\
\hline$|N|=10$ & $204(-85.8 \%)$ & $2.3 \mathrm{sec}$. & $12,253,685(-97.7 \%)$ & $11,384,820(-97.9 \%)$ \\
\hline
\end{tabular}

Table 4 - Size of Model Formulation (1)-(11), (23) for a 50-Street-Network.

\begin{tabular}{|c|c|c|c|c|}
\hline \#Cell Sizes & \#Cells & Period Length & \#Constraints & \#Variables \\
\hline$|N|=1$ & 283 & $4.0 \mathrm{sec}$. & $12,310,501$ & $12,140,700$ \\
\hline$|N|=2$ & $153(-45.9 \%)$ & $4.0 \mathrm{sec}$. & $3,692,861(-70.0 \%)$ & $3,580,200(-70.5 \%)$ \\
\hline$|N|=3$ & $107(-62.2 \%)$ & $4.0 \mathrm{sec}$. & $1,845,495(-85.0 \%)$ & $1,765,500(-85.5 \%)$ \\
\hline$|N|=4$ & $94(-66.8 \%)$ & $4.0 \mathrm{sec}$. & $1,437,362(-88.3 \%)$ & $1,367,700(-88.7 \%)$ \\
\hline$|N|=5$ & $76(-73.1 \%)$ & $4.0 \mathrm{sec}$. & $957,227(-92.2 \%)$ & $900,600(-92.6 \%)$ \\
\hline$|N|=10$ & $53(-81.3 \%)$ & $4.0 \mathrm{sec}$. & $477,001(-96.1 \%)$ & $445,200(-96.3 \%)$ \\
\hline
\end{tabular}

Table 5 - Size of Model Formulation (1)-(11), (23) for a 100-Street-Network.

\begin{tabular}{|c|c|c|c|c|}
\hline \#Cell Sizes & \#Cells & Period Length & \#Constraints & \#Variables \\
\hline$|N|=1$ & 798 & $2.7 \mathrm{sec}$. & $143,252,971$ & $142,541,154$ \\
\hline$|N|=2$ & $425(-46.7 \%)$ & $2.7 \mathrm{sec}$. & $41,032,045(-71.4 \%)$ & $40,563,700(-71.5 \%)$ \\
\hline$|N|=3$ & $292(-63.4 \%)$ & $2.7 \mathrm{sec}$. & $19,533,177(-86.4 \%)$ & $19,209,220(-86.5 \%)$ \\
\hline$|N|=4$ & $233(-70.8 \%)$ & $2.7 \mathrm{sec}$. & $12,521,443(-91.3 \%)$ & $12,262,324(-91.4 \%)$ \\
\hline$|N|=5$ & $197(-75.3 \%)$ & $2.7 \mathrm{sec}$. & $9,005,215(-93.7 \%)$ & $8,786,200(-93.8 \%)$ \\
\hline$|N|=10$ & $131(-83.6 \%)$ & $2.7 \mathrm{sec}$. & $4,031,395(-97.2 \%)$ & $3,914,542(-97.3 \%)$ \\
\hline
\end{tabular}


Table 6 - Size of Model Formulation (1)-(11), (23) for a 150-Street-Network.

\begin{tabular}{|c|c|c|c|c|}
\hline \#Cell Sizes & \#Cells & Period Length & \#Constraints & \#Variables \\
\hline$|N|=1$ & 1,432 & 2.3 sec. & $537,829,129$ & $536,334,120$ \\
\hline$|N|=2$ & $758(-47.1 \%)$ & $2.3 \mathrm{sec}$. & $151,537,691(-71.8 \%)$ & $150,554,718(-71.9 \%)$ \\
\hline$|N|=3$ & $528(-63.1 \%)$ & $2.3 \mathrm{sec}$. & $73,862,481(-86.3 \%)$ & $73,176,048(-86.4 \%)$ \\
\hline$|N|=4$ & $416(-70.9 \%)$ & $2.3 \mathrm{sec}$. & $46,035,549(-91.4 \%)$ & $45,493,344(-91.5 \%)$ \\
\hline$|N|=5$ & $345(-75.9 \%)$ & $2.3 \mathrm{sec}$. & $31,784,241(-94.1 \%)$ & $31,335,660(-94.2 \%)$ \\
\hline$|N|=10$ & $204(-85.8 \%)$ & $2.3 \mathrm{sec}$. & $11,234,485(-97.9 \%)$ & $11,021,508(-97.9 \%)$ \\
\hline
\end{tabular}

\subsection{Consideration of Lanes and Limiting Traffic Flows}

One major weakness of the CTEPM is related to the lack of explicit consideration of lanes. In its standard form, flow capacity of a cell is determined by the flow capacity per lane multiplied by the number of lanes. This might lead to problems, see Figure 3.

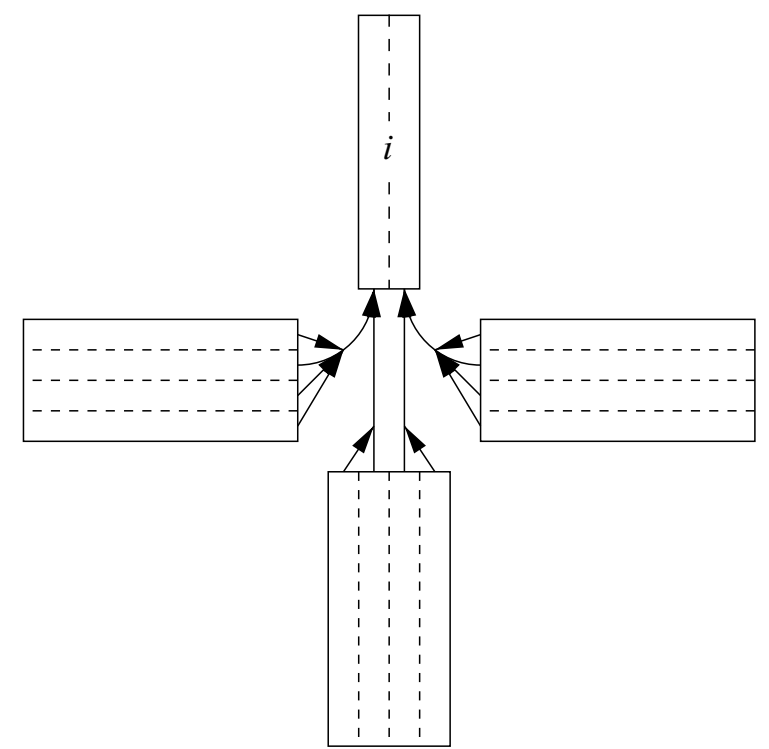

Figure 3 - Consideration of Lanes and Traffic Flow Limitations - Example.

Imagine that the total vehicle flow capacity (using all lanes) for cell $i$ (see Fig. 3) will be 16 vehicles per period. The CTEPM without explicit consideration of lanes allows that the total vehicle flow capacity per period can be divided into fractions, e.g. 6,5 and 5 vehicles per period per lane $(6+5+5=16)$. When lanes are explicitly considered in the model, vehicle flow capacity per lane would be $16 / 2=8$ vehicles per period per lane for cell $i$. Thus, vehicle flows of 6,5 and 5 vehicles per period result in $6 / 8=0.75,5 / 8=0.625$ and 0.625 lanes which will of course not be viable in real world problems. Therefore it is important to express the vehicle flow capacity per period of a cell $i$ as integer multiples of the vehicle flow capacity per lane. 
Another important aspect of safe traffic routing covers the limitation of traffic flows. Traffic flow limitation contains two essential aspects, namely merging/diverging of traffic flows as well as time-dependent traffic flow redirection. The CTEPM has no such limitations whereby solutions may be obtained where traffic flows merge and diverge very often or even will be repeatedly redirected over time. As an illustrative example, imagine a four-way junction with three fourlane streets and one two-lane street as the only available exit, see Figure 3. If a total of 12 lanes (4 lanes from each different direction) will be merged into two lanes at a junction, merging processes have to take place at the junction. Of course, this would result in a traffic chaos. However, such solutions may be optimal in a given optimization model if no corresponding constrains exist, but not in real world applications. In detail, we limit traffic routing due to three major reasons: firstly, it is not advantageous to allow an unlimited number of cells to be merged into just one cell (e.g. directly in front of a sink cell as mentioned above). Secondly, conflicting traffic flows in general as well as crossing traffic flows at junctions often exists due to multi-diverging traffic flows and thirdly, the organization of an evacuation (e.g. positioning safety fences for traffic flow routing) will be much simpler, if traffic flows are merged and diverged only when necessary and if traffic flows are not redirected over time, respectively. Such limitations can be implemented in the CTEPM by the following model formulation:

Additional Parameters:

$l_{i} \quad$ number of lanes in cell $i$

$p \quad$ maximum number of outflowing traffic streams

Additional Decision Variables:

$\varphi_{i j} \quad=1$, if traffic flows from cell $i$ to cell $j, 0$ otherwise

$\epsilon_{i j} \quad$ number of used lanes between cell $i$ and cell $j$

Given this notation the ExCTEPM with multiple cell sizes on the basis of Approach 2 can be formulated in the following manner:

$$
\min \sum_{i \in I} \sum_{t \in T} z_{i t} \cdot c_{i t}
$$

subject to (2)-(8), (11)-(16), (23) and

$$
\begin{array}{ll}
y_{i j t} \leq \frac{Q_{j t}}{l_{j}} \cdot \epsilon_{i j} & i, j \in I ; t \in T \\
y_{i j t} \leq \frac{Q_{i t}}{l_{i}} \cdot \epsilon_{i j} & i, j \in I ; t \in T \\
\sum_{i \in I} \varphi_{i j} \leq l_{j} & j \in I \\
\sum_{j \in I} \varphi_{i j} \leq p & i \in I \\
\varphi_{i j}+\varphi_{j k} \leq 1 & i, j, k \in I: \beta_{i j}=1 ; \beta_{i k}=1
\end{array}
$$




$$
\begin{array}{lc}
\varphi_{i j} \leq \beta_{i j} & i, j \in I \\
\epsilon_{i j} \leq \varphi_{i j} \cdot \min \left\{l_{i}, l_{j}\right\} & i, j \in I \\
\sum_{i \in I} \epsilon_{i j} \leq l_{j} & j \in I \\
\sum_{j \in I} \epsilon_{i j} \leq l_{i} & i \in I \\
\epsilon_{i j} \in \mathbb{N}_{0} & i, j \in I \\
\varphi_{i j} \in\{0,1\} & i, j \in I
\end{array}
$$

Due to the consideration of lanes in the ExCTEPM, constraints (9) and (10) are replaced by (25) and (26). These constraints limit the number of cell-changing vehicles to the vehicle flow capabilities of the corresponding cells under consideration of allocated lanes. Furthermore, the constraints (27)-(29) limit the traffic routing in multiple ways. (27) ensures that the number of traffic flows streaming into cell $j$ is not exceeding the number of available lanes in cell $j$. Hence merging processes at junctions due to traffic flows from different directions are not allowed any more. Constraint (28) bounds the number of outgoing traffic streams from cell $i$ to a fixed value p. A discussion of possible values for $p$ can be found in Section 4.2.

So far, the ExCTEPM may deliver solutions that are not feasible in real world applications, but in the optimization model as can be illustrated in a little example. Imagine an intersections with four entrances/exits as shown in Figures 1 and 2. If vehicle streams exist from cell $i$ to cell $k$ as well as from cell $j$ to cell $k$, cell $k$ represents a bottleneck for cell $i$ and $j$. If we further assume that the cell $j$ (or cell $i$ ) has no ingoing traffic flows, the ExCTEPM may generate a traffic stream from cell $i$ to cell $j$ that finally reaches cell $k$ to circumvent the emerging bottleneck in cell $k$. Such infeasibilities can be avoided by constraint (29) since it allows a traffic stream from cell $i$ to cell $j$ to cell $k$ to be created, if and only if no direct connection between cell $i$ and cell $k$ exist. In addition, this constraint simplifies traffic routing by forcing every street section to be used only in one direction. In addition, (30) and (31) assure that a traffic stream using a certain number of lanes from a cell $i$ to a cell $j$ only can be established, if a connection (in terms of $\beta_{i j}$ and $\varphi_{i j}$, respectively) between these cells exists. Finally, constraints (32) and (33) limit the number of used lanes between a cell $i$ and a cell $j$ to the number of available lanes regarding both cells. (34) and (35) determine the domain of the additional decision variables.

These additional constraints help to guide traffic through the network in a safer and less conflictual way since merging processes at junctions will be avoided. However, there might exist some situations where these constraints, especially constraint (27) and (32) might result in no feasible solution if the number of lanes is relatively low and many junctions exist. As an example, imagine a t-junction with three one-lane streets whereas two of these streets are deadlocks. Now, these two (deadlock) streets have to use the (non-deadlock) street in order to be evacuated, but suchlike situations are forbidden by (27) and (32). In such cases, these constraints have to be 
relaxed and a new constraint $\sum_{i \in I} y_{i j t} \leq Q_{j t}$ for all cells $j \in I$ and periods $t \in T$ must be added in order to ensure that traffic flow capacities are not exceeded.

Moreover, $\varphi_{i j}$ allows the avoidance of traffic crossing and traffic touching conflicts at junctions by formulating (36):

$$
\varphi_{i j}+\varphi_{j i}+\varphi_{k l}+\varphi_{l k} \leq 1 \quad i, j, k, l \in I
$$

Here, we assume that the cells $i, j, k, l$ adjoin to the same junction, so that $\beta_{i j}=\beta_{i k}=\beta_{i l}=$ $\beta_{j k}=\beta_{j l}=\beta_{k l}=1$ holds. Please note that this constraint also holds for different values of $p$ and for junctions with more than four entrances/exits since $i, j$ and $k, l$ pairs of cells always exist, independent of the number of entrances/exits of a junction.

\subsection{Cell Size Optimization}

Cell sizes have to be chosen adequately when a road network must be modeled as a cell network. In this section, we will introduce a simple optimization model which determines the optimum cell size(s) and the corresponding number of cells for a given road network.

Assuming a given driving speed $s$, a certain cell size always corresponds to a certain cycle time (period length) with index $k$. Thus, a cell may represent different distances depending on the chosen cycle time $k$. Table 7 shows five examples for $s=50 \mathrm{~km} / \mathrm{h}=13.89 \mathrm{~m} / \mathrm{s}$ and $s=$ $30 \mathrm{~km} / \mathrm{h}=8.33 \mathrm{~m} / \mathrm{s}$.

Table 7 - Relation between Cell Size and Cycle Time $(|N|=1)$.

\begin{tabular}{|c|c|c|c|c|c|c|}
\hline $\begin{array}{c}k \\
\text { cycle time }\end{array}$ & & $\begin{array}{c}1 \\
1.8 \mathrm{sec} .\end{array}$ & $\begin{array}{c}2 \\
3.6 \mathrm{sec} .\end{array}$ & $\begin{array}{c}3 \\
7.2 \mathrm{sec} .\end{array}$ & $\begin{array}{c}4 \\
10.8 \mathrm{sec} .\end{array}$ & $\begin{array}{c}5 \\
14.4 \mathrm{sec} .\end{array}$ \\
\hline cell size & $s=50 \mathrm{~km} / \mathrm{h}$ & $25 \mathrm{~m}$ & $50 \mathrm{~m}$ & $100 \mathrm{~m}$ & $150 \mathrm{~m}$ & $200 \mathrm{~m}$ \\
\hline cell size & $s=30 \mathrm{~km} / \mathrm{h}$ & $15 \mathrm{~m}$ & $30 \mathrm{~m}$ & $60 \mathrm{~m}$ & $90 \mathrm{~m}$ & $120 \mathrm{~m}$ \\
\hline
\end{tabular}

As can be easily derived from Table 7, smaller cycle times are leading to smaller cell sizes, which enable us to represent a given network very accurate, by trend, but usually a larger number of (small) cells is required due to the lower cell size.

If we are using multiple cell sizes as explained in Section 2.1, every cycle time $k$ corresponds to $|N|$ different cell sizes for a given driving speed $s$. Moreover, we assume every cell size of a given cycle time $k$ to be a corresponding "version" $l$ of this certain cycle time $k$ with a length of $g_{k l}$ so that, for example, a cycle time $k$ leads to (for $|N|=3$ and two different driving speeds) six versions with a length of $\left(g_{k 1}, g_{k 2}, g_{k 3}, g_{k 4}, g_{k 5}, g_{k 6}\right)$. This notation has to be interpreted in the following manner:

\begin{tabular}{|c|c|}
\hline Cells at $50 \mathrm{~km} / \mathrm{h}$ driving speed & Cells at $30 \mathrm{~km} / \mathrm{h}$ driving speed \\
\hline$g_{k 3}$ & $g_{k 4}$, \\
\hline
\end{tabular}


Due to the discrete nature of the ExCTEPM, the assumption that vehicles drive at $50 \mathrm{~km} / \mathrm{h}$ and $30 \mathrm{~km} / \mathrm{h}$ and taking $g_{k 1}$ (single cell size, $50 \mathrm{~km} / \mathrm{h}$ driving speed) as a point of reference, the following relations between these six versions exist:

$$
\begin{aligned}
& g_{k 2}=2 \cdot g_{k 1} \\
& g_{k 3}=3 \cdot g_{k 1} \\
& g_{k 4}=0.6 \cdot g_{k 1} \\
& g_{k 5}=0.6 \cdot g_{k 2}=1.2 \cdot g_{k 1} \\
& g_{k 6}=0.6 \cdot g_{k 3}=1.8 \cdot g_{k 1}
\end{aligned}
$$

Given these considerations, Table 7 can be complemented as shown in Table 8:

Table 8 - Relation between Cell Size and Cycle Time $(|N|=3)$.

\begin{tabular}{|c|c|c|c|c|c|c||c|}
\hline $\begin{array}{c}k \\
\text { cycle time }\end{array}$ & & $\begin{array}{c}1 \\
1.8 \mathrm{sec} .\end{array}$ & $\begin{array}{c}2 \\
3.6 \mathrm{sec} .\end{array}$ & $\begin{array}{c}3 \\
7.2 \mathrm{sec} .\end{array}$ & $\begin{array}{c}4 \\
10.8 \mathrm{sec} .\end{array}$ & $\begin{array}{c}5 \\
14.4 \mathrm{sec} .\end{array}$ & $l$ \\
\hline cell size $\left(I_{1}\right)$ & $s=50 \mathrm{~km} / \mathrm{h}$ & $25 \mathrm{~m}$ & $50 \mathrm{~m}$ & $100 \mathrm{~m}$ & $150 \mathrm{~m}$ & $200 \mathrm{~m}$ & 1 \\
\hline cell size $\left(I_{2}\right)$ & $s=50 \mathrm{~km} / \mathrm{h}$ & $50 \mathrm{~m}$ & $100 \mathrm{~m}$ & $200 \mathrm{~m}$ & $300 \mathrm{~m}$ & $400 \mathrm{~m}$ & 2 \\
\hline cell size $\left(I_{3}\right)$ & $s=50 \mathrm{~km} / \mathrm{h}$ & $75 \mathrm{~m}$ & $150 \mathrm{~m}$ & $300 \mathrm{~m}$ & $450 \mathrm{~m}$ & $600 \mathrm{~m}$ & 3 \\
\hline \hline cell size $\left(I_{1}\right)$ & $s=30 \mathrm{~km} / \mathrm{h}$ & $15 \mathrm{~m}$ & $30 \mathrm{~m}$ & $60 \mathrm{~m}$ & $90 \mathrm{~m}$ & $120 \mathrm{~m}$ & 4 \\
\hline cell size $\left(I_{2}\right)$ & $s=30 \mathrm{~km} / \mathrm{h}$ & $30 \mathrm{~m}$ & $60 \mathrm{~m}$ & $120 \mathrm{~m}$ & $180 \mathrm{~m}$ & $240 \mathrm{~m}$ & 5 \\
\hline cell size $\left(I_{3}\right)$ & $s=30 \mathrm{~km} / \mathrm{h}$ & $45 \mathrm{~m}$ & $90 \mathrm{~m}$ & $180 \mathrm{~m}$ & $270 \mathrm{~m}$ & $360 \mathrm{~m}$ & 6 \\
\hline
\end{tabular}

It is now clear that the optimization of cell sizes is equivalent to the selection of the optimum cycle time, whereas conflicting objectives must be handled: on the one hand, cell sizes of a cycle time $k$ should be as small as necessary to match the corresponding arc length perfectly, but on the other hand, cell sizes of this cycle time $k$ should be as big as possible so that only few cells are needed and that the number of variables and constraints in the CTEPM and ExCTEPM can be kept low.

In order to represent a given network adequately, it is useful to determine a certain level of accuracy, i.e. for example the maximum deviation between the length of a street section and the cumulated length(s) of cell(s) assigned to this street section. Hence, the following MIP model selects the optimum cycle time in order to minimize the number of cells which are required to represent the network under consideration of the predetermined level of accuracy.

Please note that the standard CTEPM is a special case of the ExCTEPM, if traffic flow limitations are not considered. As such, the presented model can be used for the CTEPM as well. We use the following notation for the optimization model: 


\section{Parameters:}
$K=\{1, \ldots,|K|\} \quad$ Index set of cycle times
$S=\{1, \ldots,|S|\} \quad$ Index set of driving speeds
$J_{S} \quad$ Index set of street sections at driving speed $s$
$J=\bigcup_{s \in S} J_{S}$
$P_{S}$
Index set of street sections
Index set of available versions which can be used at street sections at driving speed $s$
$P=\bigcup_{s \in S} P_{S}$
Index set of available versions
$g_{k l}$
length $[\mathrm{m}]$ of version $l$ of cycle time $k$
$d_{j}$
length $[\mathrm{m}]$ of street section $j$
$o_{j}$
maximum relative deviation between the length of street
C section $j$ and the (cumulated) length(s) of the corresponding cells maximum number of cells

Decision Variables:

$\beta_{k j l} \quad$ number of used cells of version $l$ and cell type $k$ at street section $j$

$\gamma_{k} \quad=1$, if cell type $k$ is used, 0 otherwise

$Z_{j} \quad$ difference $[\mathrm{m}]$ between the length of street section $j$ and the intended cell(s)

$$
\min \sum_{k \in K} \sum_{j \in J} \sum_{l \in P} \beta_{k j l}
$$

subject to

$$
\begin{array}{ll}
\sum_{j \in J} \sum_{l \in P} \beta_{k j l} \leq \gamma_{k} \cdot C & k \in K \\
\sum_{k \in K} \sum_{l \in P} \beta_{k j l} \geq 1 & j \in J \\
\sum_{k \in K} \gamma_{k}=1 & \\
\sum_{k \in K} \beta_{k j l}=0 & s \in S ; j \in J_{s} ; l \in P_{h: h \neq s} \\
d_{j}-\sum_{k \in K} \sum_{l \in P} g_{k l} \cdot \beta_{k j l} \leq Z_{j} & j \in J \\
d_{j}-\sum_{k \in K} \sum_{l \in P} g_{k l} \cdot \beta_{k j l} \geq-Z_{j} & j \in J \\
Z_{j} \leq o_{j} \cdot d_{j} & \\
\beta_{k j l} \in \mathbb{N}_{0} & k \in J \\
\gamma_{k} \in\{0,1\} & k \in K ; j \in J ; l \in P \\
Z_{j} \geq 0 & k \in K
\end{array}
$$


The objective (37) is to minimize the total number of required cells. Constraint (38) ensures that a version $l$ of cycle time $k$ only can be used, if the corresponding cycle time is chosen to be used. To avoid infeasible solutions for a given set of cycle times due to too small values for $C$ (Constraint (39) forces every street section to be covered at least by one cell), a lower bound for $C$ can be calculated by (48):

$$
C \geq \sum_{s=1}^{|S|} \sum_{j \in J_{s}}\left\lceil\frac{d_{j}}{\max _{k \in K, l \in P_{s}} g_{k l}}\right\rceil
$$

Equation (40) fixes the number of used cycle times to one and equation (41) assures that no inappropriate versions of cycle times are used. Constraint (42) as well as (43) calculate the actual difference between the length of street section $j$ and the length(s) of the corresponding cells. An upper bound for this deviation is stated in (44). Finally, the domains of the decision variables are stated in (45), (46) and (47).

Beside the objective stated in (37), other objectives are also implementable. For example, the minimization of total deviation for a given $C$ can be integrated by dropping constraint (44) and replacing the objective function (37) with (49)

$$
\min \sum_{j \in J} Z_{j}
$$

For a given maximum number of cells this objective function will minimize the sum of all deviations between the length of street section $j$ and the cell(s) intended to model this street section. The lowest possible objective value is $\sum_{j \in J} Z_{j}=0$, i.e. all cells match perfectly the given street sections.

Another objective may cover the minimization of the largest deviation between a certain street section $j$ and the corresponding cells. This objective can be implemented by replacing (49) with (50)

$$
\min W
$$

and adding constraint (51):

$$
W \geq Z_{j} \quad j \in J
$$

In this case, $W$ will be set to the largest $Z_{j}$ value whereas $Z_{j}$ denotes the deviation between the length of street section $j$ and the cell(s) intended to model this street section in the ExCTEPM.

If the (total/largest) deviation is minimized, additional constraints for automatic selection of large cell sizes (where possible) can eliminate redundancy, see (52):

$$
\sum_{k \in K} \sum_{L \in \mathcal{P}\left(L_{S}\right)} \sum_{l \in L} \beta_{k j l} \leq 1 \quad s \in S ; j \in J_{S} ; L_{S}=\left\{1, \ldots,\left|P_{S}\right|-1\right\}
$$




\subsection{Numerical Example}

In order to illustrate the effect of multiple cell sizes as well as the explicit consideration of lanes and traffic flow limitations, we will present a numerical example in this section. In particular, four different cases will be computed:

1. Case: CTEPM with single cell size: (1)-(16).

2. Case: CTEPM with multiple cell sizes (Approach 1): (1), (4)-(16), (17)-(22).

3. Case: CTEPM with multiple cell sizes (Approach 2): (1)-(16), (23).

4. Case: CTEPM with single cell size and consideration of lanes and traffic flow limitations: (1)-(8), (11)-(16) and (25)-(36).

5. Case: CTEPM with multiple cell sizes (Approach 1), consideration of lanes and traffic flow limitations: (1), (4)-(8), (11)-(16), (17)-(22) and (25)-(36).

6. Case: CTEPM with multiple cell sizes (Approach 2), consideration of lanes and traffic flow limitations (=ExCTEPM):(1)-(8), (11)-(16), (23) and (25)-(36).

The considered test network consists of straight street sections of different lengths as displayed in Figure 4. The driving speed is $13.89 \mathrm{~m} / \mathrm{s}$ on all $50 \mathrm{~m} / 100 \mathrm{~m}$ sections and $8.33 \mathrm{~m} / \mathrm{s}$ on all $30 \mathrm{~m} / 60 \mathrm{~m}$ sections. Using a period length of 3.6 seconds, the resulting cell sizes are $13.89 \mathrm{~m} / \mathrm{s} \cdot 3.6 \mathrm{~s}=50 \mathrm{~m}$ and $8.33 \mathrm{~m} / \mathrm{s} \cdot 3.6 \mathrm{~s}=30 \mathrm{~m}$. All cells are two- or four-laned. This network can be modeled in the single cell size CTEPM with 51 cells (including super sink), see Figure 5. If we assume that multiple cell sizes can be used, larger cells of $60 \mathrm{~m} / 100 \mathrm{~m}$ can be defined, so that two adjacent cells on a street section can be replaced by one larger cell. Now, the same network can be modeled with 35 cells (cell 1-18 single sized, cell 19-34 double sized and a super sink), see Figure 6. In this case, cell sizes of $50 \mathrm{~m} / 100 \mathrm{~m}$ and $30 \mathrm{~m} / 60 \mathrm{~m}$ match the street section lengths of the given road network perfectly. We set $c_{i t}=c_{i} \cdot t$ with $c_{i}=100$ for all cells except the super sink $\left(c_{i}=0\right.$ for the super sink) and $|T|$ is set to $|T|=1.5 \cdot \sum_{i \in I} E_{i} / \sum_{i \in I_{S}} Q_{i}=1.5 \cdot 2422 / 54 \approx 67$.

The computation times for solving the LPs (Case 1,2,3) optimally and MIPs (Case 4,5,6) to a maximum mipgap of $0.1 \%$ are shown in Table 9 .

Table 9 - Computation Times for Random Network with One and Two Cell Sizes.

\begin{tabular}{|l|c|c|c|}
\hline Model & $\begin{array}{c}\text { Computation Time } \\
\text { [sec.] }\end{array}$ & $\begin{array}{c}\text { \#Constraints } \\
\text { (after AMPL's Presolve) }\end{array}$ & $\begin{array}{c}\text { \#Variables } \\
\text { (after AMPL's Presolve) }\end{array}$ \\
\hline 1. Case & 5.3 & 19,914 & 22,960 \\
\hline 2. Case & 3.1 & 14,699 & 18,834 \\
\hline 3. Case & 2.4 & 14,683 & 17,746 \\
\hline \hline 4. Case & 1919.6 & 40,056 & 23,352 \\
\hline 5. Case & 876.1 & 32,668 & 19,096 \\
\hline 6. Case & 505.8 & 32,652 & 18,008 \\
\hline
\end{tabular}




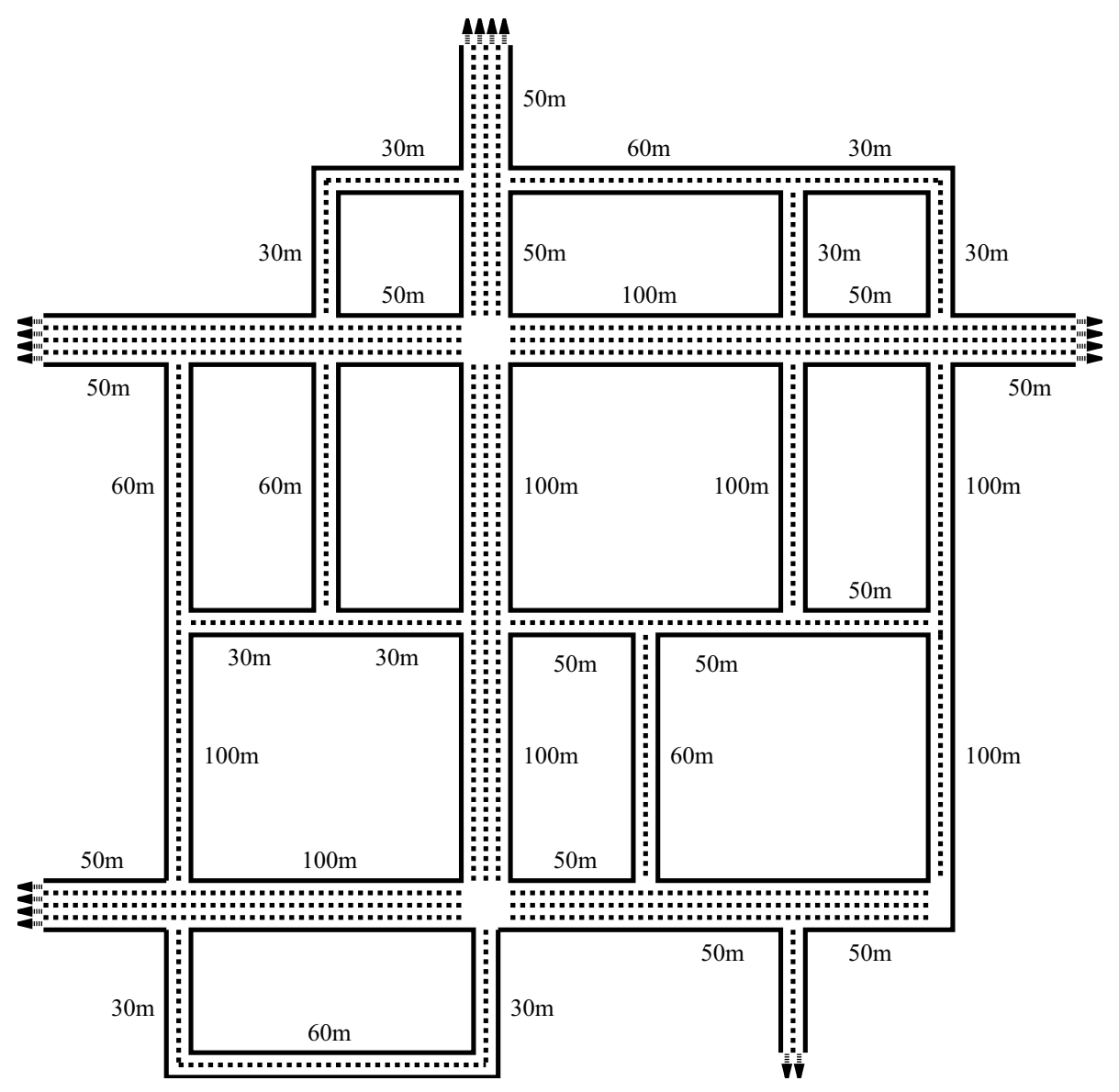

Figure 4 - Random Street Network.

The CTEPM and the CTEPM with multiple cell sizes are linear programs (LP) which can be solved very fast using by GUROBI's (see http://www.gurobi.com) simplex algorithm (Case 1, 2, 3). Implementing lanes and traffic flow limitations into the model formulation results in a mixed integer problem (MIP) due to the introduction of $\varphi_{i j}$ and $\epsilon_{i j}$, so that the GUROBI's branch \& bound algorithm must be applied (Case 4, 5,6). This leads to much larger computation times compared to the LPs. For all MIPs, GUROBI finds good (mipgap $\leq 0.1 \%$ ) feasible solutions very fast. Using multiple cell sizes helps to reduce the computation time significantly. In particular, computation time drops by $41.5 \% / 54.7 \%$ for the LPs and $54.4 \% / 73.6 \%$ for the MIPs. It should be mentioned that the application of multiple cell sizes reduces the number of cells by $31 \%$ in this case. For other instances (with larger reduction of cells due to multiple cell sizes), results may vary.

Another important aspect is the implementation of different driving speeds into the model. Of course, any number of different driving speeds can be modeled in all model formulations. Let us consider the random street network (Fig. 4) again. For the sake of simplicity, we may assume 


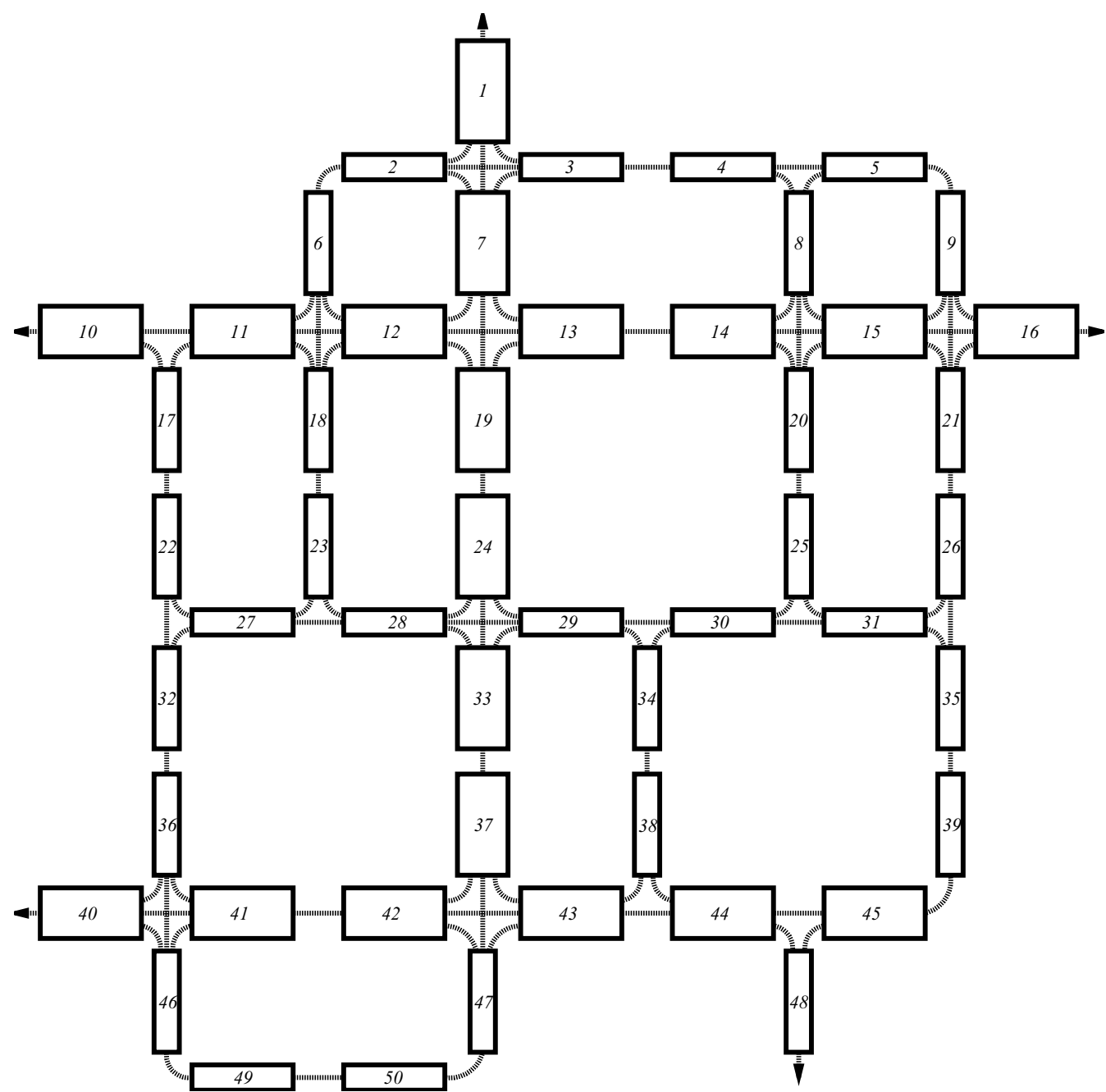

Figure 5 - Random Street Network Representation with One Cell Size.

that the driving speed corresponds to the length of the street section, so that street section lengths of $30 \mathrm{~m}|50 \mathrm{~m}| 60 \mathrm{~m} \mid 100 \mathrm{~m}$ lead to driving speeds of $30 \mathrm{~km} / \mathrm{h}|50 \mathrm{~km} / \mathrm{h}| 60 \mathrm{~km} / \mathrm{h} \mid 100 \mathrm{~km} / \mathrm{h}$. The corresponding cell network will look identical to the case of using two cell sizes in Figure 6, but there are significant differences in the model formulation since now only single cell sizes with doubled driving speed (instead of doubled cell sizes with standard driving speed) are used. Therefore, all larger cells can be passed by one period instead of two periods. However, subsequent adjustments of driving speeds may require different cell sizes which need to be defined in a way to match the given street lengths adequately. In summary, any number of driving speeds can be combined with any number of different cell sizes. We will present an approach to determine the “optimum" cell sizes in the Section 4.2. 


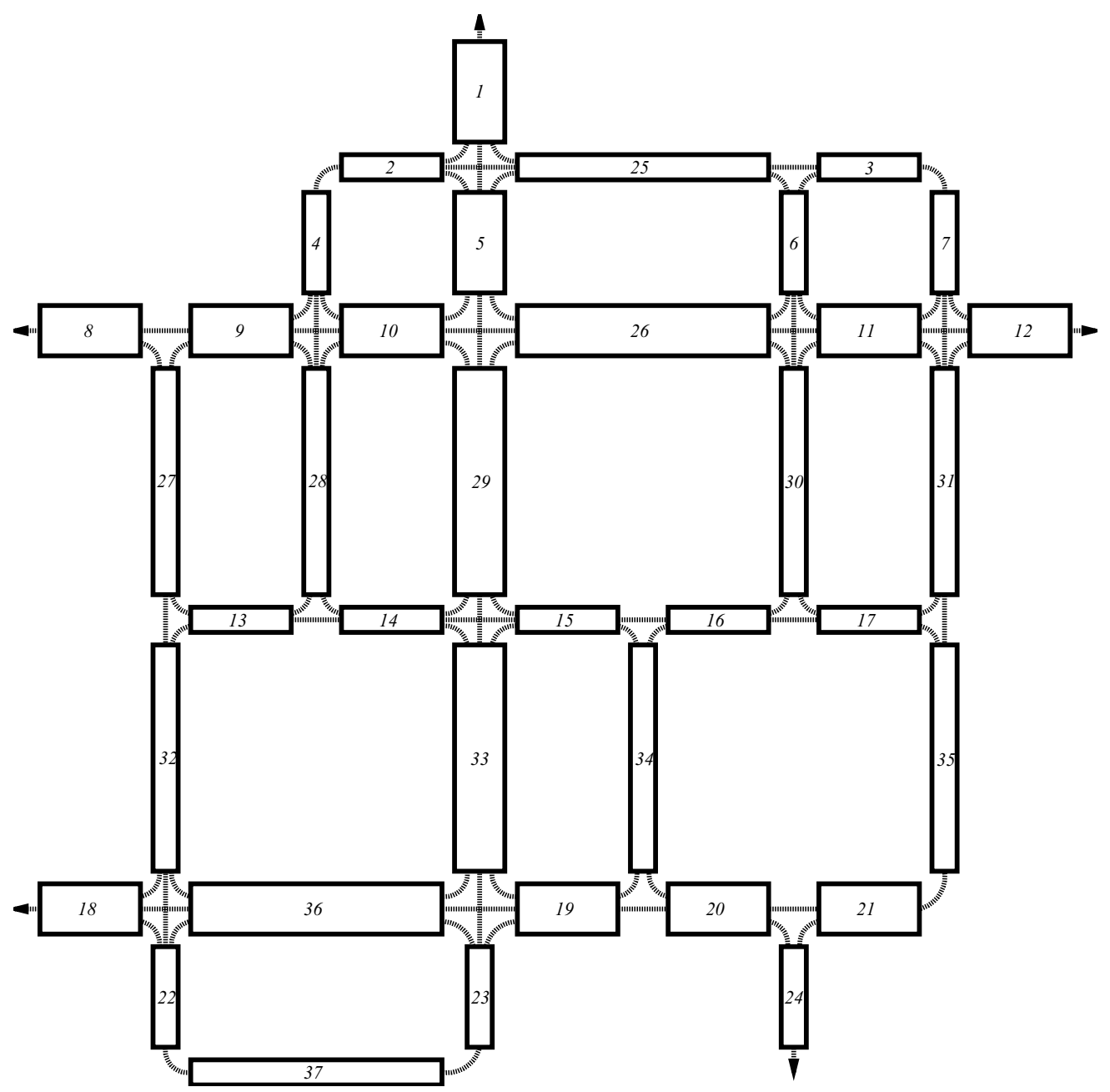

Figure 6 - Random Street Network Representation with Two Cell Sizes.

\section{SIMULATION-BASED OPTIMIZATION APPROACH}

When it comes to evacuation planning problems or traffic assignment problems in general, complex and dynamic traffic behavior is very hard to capture in an optimizational environment so that traffic simulations are the method of choice to evaluate solutions, see [9, 15, 21, 25]. Since there might exist a gap between the solution of the ExCTEPM and its implementation in a microscopic traffic simulation, we will use the observations gathered from the traffic simulation to modify the the evacuation routing in such a way that the outcome of the traffic simulation approximates the outcome of the optimization model in terms of exit occupation times.

We choose the exit occupation time as the key figure since for our real world example the ExCTEPM tends to generate solutions where the exit occupation times are well balanced. This result indicates that unused flow capacities at exits due to unbalanced exit occupation times 
have to be avoided. It has to be mentioned that similar exit occupation times may lead to different number of vehicles escaping through these exits because of different flow capacities, e.g. different number of lanes. Admittedly, the optimal solution of the ExCTEPM for extremely sparse networks may not result in perfectly balanced exit occupation times since traffic flow limitations may force some exits to be used only by a small number of vehicles. However, we assume that such affects will not appear in urban areas since urban street networks are often strongly connected.

We will discuss the components (reallocation of vehicles and traffic flow limitations) as well as the simulation-based optimization procedure itself in the following:

Vehicle Reallocation Model. The ExCTEPM allows to optimize traffic routing in road networks under consideration of queuing effects on a mesoscopic level of detail. We will now introduce a reallocation optimization model which will use data provided by our microscopic traffic simulation (SUMO). In particular, we assume a district $i$ to be defined as the set of cells escaping to exit $i$ so that the number of districts is equal to the number of exits. We imply that all districts are part of a connected network, i.e. there are no insurmountable obstacles between districts, e.g. no options to cross a river by bridges or ferry boats. It has to be made clear that the reallocation model does not determine the shape of the districts themself, because the shape of the districts is the outcome of the ExCTEPM. We formulate the model using the following notation:

\section{Parameters:}

$D$ Index set of district/exits

$s_{i} \quad$ number of vehicles using exit $i$ (before reallocation)

$p_{i} \quad$ largest index of period where exit $i$ is still occupied (before reallocation)

Decision Variables:

$w_{i j} \quad$ number of vehicles reallocated from exit $i$ to exit $j$

$e_{i} \quad$ number of vehicles using exit $i$ (after reallocation)

$t_{i} \quad$ largest index of period where exit $i$ is still occupied (after reallocation)

$Z \quad$ auxiliary variable

Based on this notation, the reallocation model can be formulated as a MIP model by:

$$
\min Z
$$

subject to

$$
\begin{array}{ll}
Z \geq t_{i} & i \in D \\
e_{i}=s_{i}+\sum_{j \in I} w_{j i}-\sum_{j \in I} w_{i j} & i \in D \\
\frac{s_{i}}{p_{i}}=\frac{e_{i}}{t_{i}} & i \in D
\end{array}
$$




$$
\begin{array}{ll}
w_{i j} \in \mathbb{N}_{0} & i, j \in D \\
e_{i} \in \mathbb{N}_{0} & i \in D \\
t_{i} \geq 0 & i \in D \\
Z \geq 0 &
\end{array}
$$

The objective function (53) in combination with constraint (54) minimizes the largest occupation time of all exits. Thus, the reallocation model determines the number of vehicles escaping through a certain exit in such a way that the overall occupation time for all exits will be balanced. In equation (55) the number of vehicles allocated to exit $i$ (after reallocation) is calculated by the number of vehicles using exit $i$ before reallocation plus the number of vehicles using exit $i$ instead of exit $j$ minus the number of vehicles using exit $j$ instead of exit $i$. In addition, equation (56) approximates the index of period where exit $i$ is not occupied any more after reallocation. Here, we assume that the throughput is constant over time so that the relation between escaped vehicles and the number of occupied periods is linear. Lastly, (57)-(60) determine the domains of the decision variables.

Additional Traffic Flow Limitations. It may happen that junctions are too small so that a large number of different traffic flows may affect each other in terms of traffic blocking. Figure 7 displays such a case for a four way junction where traffic from cell $i$ to cell $u$ may be blocked due to not enough space on the junction.

Suchlike situations are forbidden by (36) so that these effects will not take place when the ExCTEPM is solved. However, constraint (36) makes the ExCTEPM also very hard to solve so that a relaxation of this constraint is likely to be used within a heuristic procedure. As will be seen later in this section, we will solve the ExCTEPM with a relaxed version of (36) that only covers traffic crossing, but not traffic touching conflicts like shown in Figure 7. If it comes to solutions where traffic is blocked due to missing space on a junction, the original constraint (36) will be restored for this junction.

Simulation-Based Optimization Procedure. We can use the vehicle reallocation model and additional traffic flow limitations to create an iterative solution heuristic composed of the these approaches, the ExCTEPM and the microscopic traffic simulation. The procedure works in the following manner:

1. Solve the ExCTEPM ((1)-(8), (11)-(16), (23), (25)-(36)).

2. Evaluation of this solution using the simulation model. (Result: Occupation time for each exit, number of vehicles at each exit and traffic blocking if so).

3. Stop, if stopping criterion is reached. (Given number of iterations without improvements.)

4. If total evacuation time is elongated due to blocked traffic streams on a junction, restore the original version of (36) for this junction and go back to Step 1. 


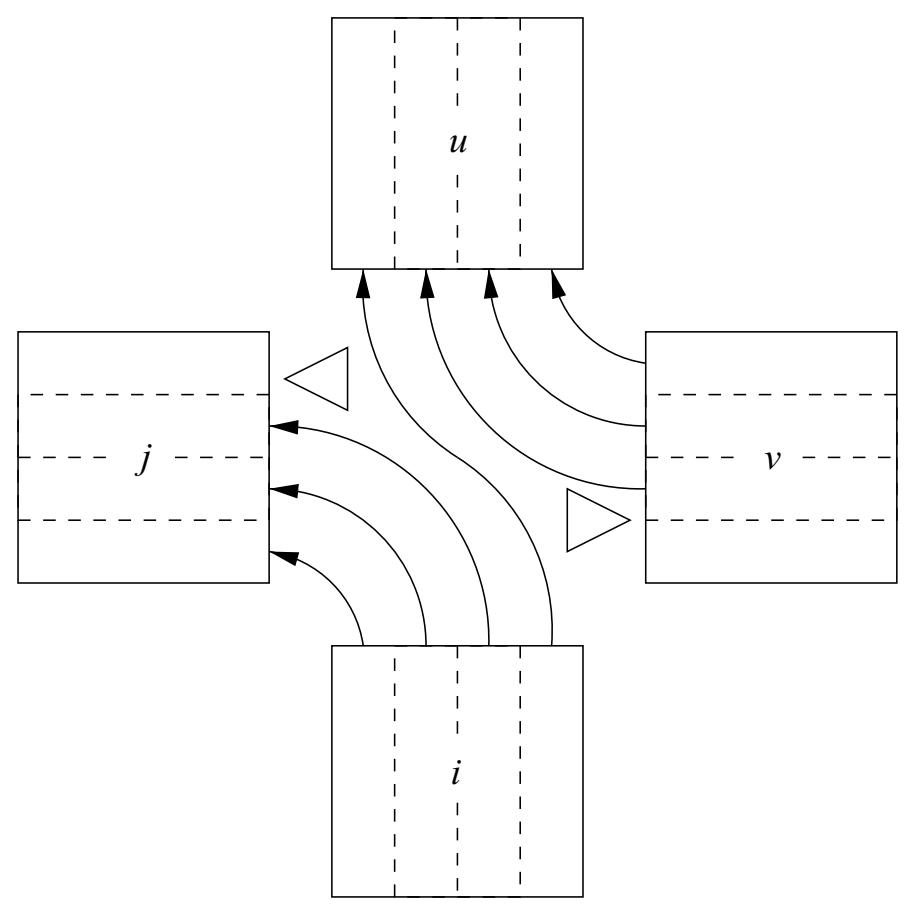

Figure 7 - Possible Routing at a Junction.

5. Solve the vehicle reallocation model ((53)-(60)) with the data from Step 2. Stop, if relative reallocation is lower than a given percentage on all exit cells.

6. Set for all $i \in I_{S}: \sum_{t \in T} y_{i,|I|, t} \leq\left\lceil(1+\mu) \cdot e_{i}\right\rceil$.

7. Fix routing decisions (number of lanes $\left(\epsilon_{i j}\right)$ between two cells) for all cells $i \in I \backslash I_{S}$ representing more than $1 \%$ of total traffic (disregarding traffic to the super sink).

8. Return to Step 1.

Rounding in Step 6 is introduced to limit traffic flow of exit cells by an integer number of vehicles. Otherwise, the optimization model tends create solutions with non-integer values for $y_{i j t}$ which are not suitable in the SUMO traffic simulation. Step 7 is inserted to ensure that the most relevant traffic routing decisions stay the same when the reallocation model is applied. Otherwise, other traffic routing decisions may lead to different traffic throughput in highly frequented cells which are not covered in the reallocation model. Fixing these decisions also results in less computation time as will be seen later in this section. In addition, $\mu \geq 0$ is required to allow a certain degree of freedom in traffic routing. We set $\mu=0.025$ in our computational study.

\section{COMPUTATIONAL STUDY}

For our computational study, the ExCTEPM and the vehicle reallocation model were coded in AMPL (see http://www.ampl.com) and solved with GUROBI 3.0.1 on a computer using an Intel X9100 (3.06 Ghz), 8 GB Memory and Windows Vista (64-bit). 


\subsection{Scenario Basics}

We tested our simulation-based optimization procedure using data from the neighborhood Neudorf in Duisburg, Germany, with a population of around 26,000 residents leading to 8,750 vehicles. The road network consists of one-, two- and four-lane streets at $50 \mathrm{~km} / \mathrm{h}$ and $30 \mathrm{~km} / \mathrm{h}$. In addition, eight exits can be used for evacuation purposes. Figure 8 shows an aerophoto of Neudorf including population density and available exits.

We decided that it is not effective to include every small side- and dead-end street in the ExCTEPM. As such we disregard small and sparsely populated street sections so that the considered street network consists of 98 street sections with a total length of $23 \mathrm{~km}$ plus eight exit street sections (and a super sink).

For all tests, we use an evacuation scenario where $100 \%$ of residents have to be evacuated. Addionally, we assume that there is no initial network traffic (e.g. due to rush hour traffic) and that departure times of all residents can be described by a gamma distribution, see (61).

$$
F(\tilde{x})=1-e^{-\tilde{x} / \beta} \sum_{j=0}^{\alpha-1} \frac{(\tilde{x} / \beta)^{j}}{j !} \quad \forall \tilde{x} \geq 0 ; \alpha \in \mathbb{N}
$$

Although the departure pattern in the case of an evacuation is not very well explored in literature, we decided to use a gamma distribution since two pertinent works by [14] and [29] confirm our assumption. Moreover, we decided to use $\alpha=3$ and $\beta=1$ since these parameter settings are reasonable to show the benefits of improved traffic routing which may be diluted, if vehicles are departing too slow, e.g. $\alpha=3 ; \beta=3$ or $\alpha=3 ; \beta=5$.

\subsection{Optimization Basics}

To calculate the optimum cell size, we first have to determine what an optimum cell size is. We will discuss three ideas in the following:

1. Minimum number of cells: In this case, each street section has to be modeled by exactly one cell, where cells can have different sizes. $\mathrm{X}$

2. Maximum accuracy in network representation: Here, the standard cell size has to be very small in order to match the lengths of the street sections adequately. Again, multiple cell sizes can be used.

3. Shortest computation time: Number of cells and cell sizes have to be chosen in way that minimizes computation time.

The minimum number of cells is determined by the number of street sections. Here, the standard cell size negatively correlates with the accuracy in network representation, i.e. higher accuracy leads to smaller standard cell sizes by trend. Smaller cells result in shorter periods so that the number of periods to cover a certain planning horizon increases. The maximum accuracy in network representation is achieved, when all cells (of different size) match the corresponding 


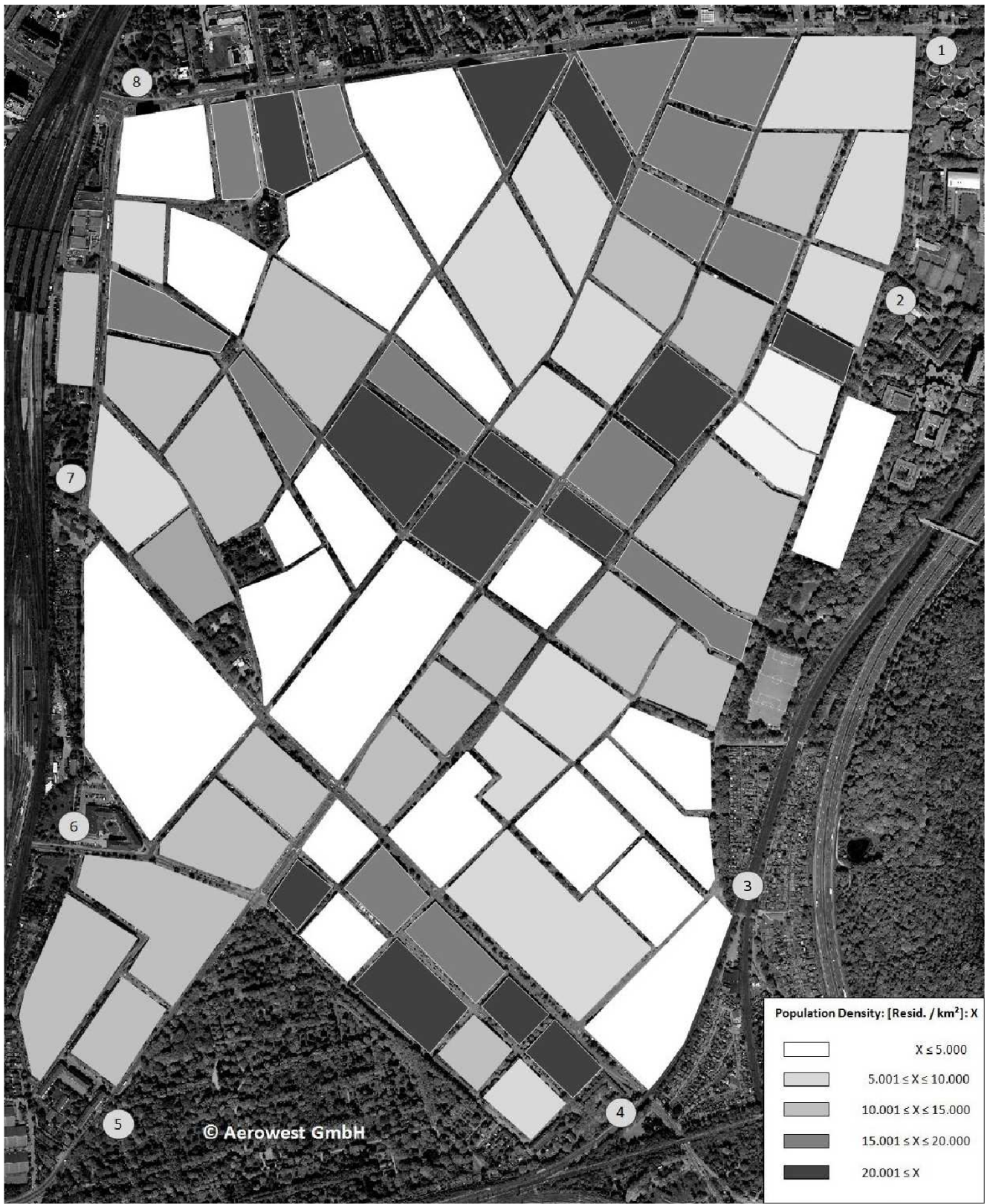

Figure 8 - Population Density and Exits in Neudorf.

street sections perfectly $\left(o_{j}=0 \%\right.$ for all $\left.j \in J\right)$. However, in extreme situations this may lead to cell sizes of about $1 \mathrm{~m}$ which results in very high number of periods due to short periods. These considerations should demonstrate that the problem size depends on the number of cells and the size of the cells which directly affects the number of periods. However, it is very hard to determine the effects to an increasing number of cells and periods so that the minimization of 
computation time by finding an "optimal" trade-off between number of cells, size of cells and accuracy in network representation is very difficult.

For our computational tests, we decide to minimize the number of cells. The number of different cell sizes directly corresponds to the accuracy in network representation. We run a short sensitivity analysis to capture these effects, see Table 10 .

We use the model (37)-(47) presented in Section 2.3 with a slightly modified objective function. In detail, we added $+0.0001 \cdot \sum_{j \in J} Z_{j}$ to the objective function. By doing so, the gap between the length of a street section and the corresponding cell(s) will be minimized, as long as no further cells are needed. This modification helps to reduce total deviation when multiple cell sizes are used, because it may be beneficial to model a certain street by a cell of size $n=5$ instead of size $n=4$, whereas both cell sizes may be feasible in terms of (44). We set the accuracy level $o_{j}=0.1,0.15, \ldots, 0.35,0.4,|K|=100$ and $|S|=2(50 \mathrm{~km} / \mathrm{h}$ and $30 \mathrm{~km} / \mathrm{h})$. First, we calculate the number of cells with a single cell size for every driving speed for a given accuracy. By doing so, we can derive the minimum (multiple) cell size desired to describe every street section by one cell by $\max _{k \in K, j \in J, l \in P} \beta_{k j l}$. In a second run, we use the (minimum) multiple cell sizes for each level of accuracy to model each street section by exactly one cell. Table 10 contains some key figures of the sensitivity analysis.

Table 10 - Sensitivity Analysis for Different Levels of Accuracy.

\begin{tabular}{|c|c|c|c|c|c|}
\hline$o_{j}$ values & Cell size $(50 \mathrm{~km} / \mathrm{h})$ & $|N|$ & Period length & Mean Deviation & Total Deviation \\
\hline $10 \%$ & $30 \mathrm{~m}$ & 25 & $2.2 \mathrm{~s}$ & $6.7 \mathrm{~m}$ & $661 \mathrm{~m}$ \\
\hline $15 \%$ & $44 \mathrm{~m}$ & 16 & $3.2 \mathrm{~s}$ & $10.8 \mathrm{~m}$ & $1060.2 \mathrm{~m}$ \\
\hline $20 \%$ & $52 \mathrm{~m}$ & 13 & $3.7 \mathrm{~s}$ & $10.8 \mathrm{~m}$ & $1061.4 \mathrm{~m}$ \\
\hline $25 \%$ & $70 \mathrm{~m}$ & 9 & $5.0 \mathrm{~s}$ & $15.8 \mathrm{~m}$ & $1547 \mathrm{~m}$ \\
\hline $30 \%$ & $82 \mathrm{~m}$ & 7 & $5.9 \mathrm{~s}$ & $19.4 \mathrm{~m}$ & $1898.2 \mathrm{~m}$ \\
\hline $35 \%$ & $106 \mathrm{~m}$ & 5 & $7.6 \mathrm{~s}$ & $27.2 \mathrm{~m}$ & $2669.4 \mathrm{~m}$ \\
\hline $40 \%$ & $122 \mathrm{~m}$ & 4 & $8.8 \mathrm{~s}$ & $32.5 \mathrm{~m}$ & $3185 \mathrm{~m}$ \\
\hline
\end{tabular}

The problem size of the ExCTEPM mainly depends on the number of cells and the number of periods. As the number of cells is the same for all accuracy levels in Table 10, problem size is only up the number of periods which correlates the standard cell size. We have computed solutions for the case of $o_{j}=0.25,0.3,0.35,0.4$ using the heuristic procedure later in this section to demonstrate the effect of increased problem size (due to more periods). The computation times are stated in Table 11.

On the basis that the ExCTEPM must be solved several times in the simulation-based optimization approach in Section 3, we decided to set $o_{j}=0.35$ since this level of accuracy is a good compromise between computation time and level of accuracy.

For adjacent cells $i$ and $j$ with equal level of danger $\left(c_{i t}=c_{j t}\right)$, the ExCTEPM (and CTEPM) tends to generate solutions, where vehicles commute between these cells. This phenomenon does not correspond to a desired "straight-forward" solution. Therefore, the term $+10^{-5}$. 
Table 11 - Computation Times for Different Levels of Accuracy.

\begin{tabular}{|c|c|}
\hline$o_{j}$ values & Computation Time [sec.] \\
\hline $25 \%$ & 11,554 \\
\hline $30 \%$ & 15,578 \\
\hline $35 \%$ & 4,318 \\
\hline $40 \%$ & 2,885 \\
\hline
\end{tabular}

$\sum_{i \in I} \sum_{j \in I} \sum_{t \in T} y_{i j t}$ has been added to the objective function (24) to avoid unnecessary vehicle movements. Moreover, a second term $-10^{-6} \cdot \sum_{t \in T} x_{|I|, t}$ has been added to the objective in order to ensure that evacuating vehicles starting in sink cells are not evacuating later than necessary. This may happen when sink cells and the super sink share the same level of danger $\left(c_{i t}\right)$ so that there is no difference between residing in a sink cell or the super sink. During our computational tests, we also found out that the second term helps to reduce computation time significantly, so that it will be kept for our computational study. Furthermore, we assume that $c_{i t}=c_{i} \cdot t$ holds and that danger is distributed uniformly within the actual network, setting $c_{i}=100$ for all "regular" cells and $c_{i}=0$ for the super sink.

Because of (33), different values for $p$ will only affect cells with $l_{i}>p$ so that only values of $p=\left\{1,2, \ldots, \max _{i \in I} l_{i}\right\}$ are useful. For this case study, we set $p=2$ since a large amount of cells is one- or two-laned $\left(l_{i}=\{1,2\}\right)$. For cells with four lanes, setting $p=2$ will provide advantages in evacuation organization, see Figure 9 and 10.

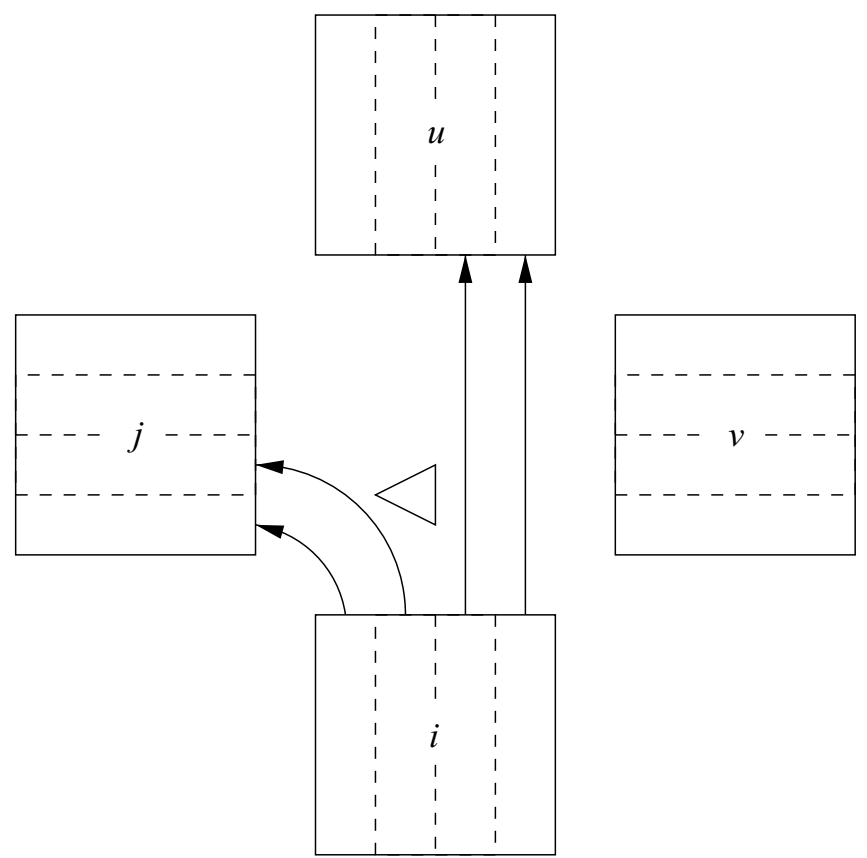

Figure 9 - Possible Routing at Intersection for $p=2$. 


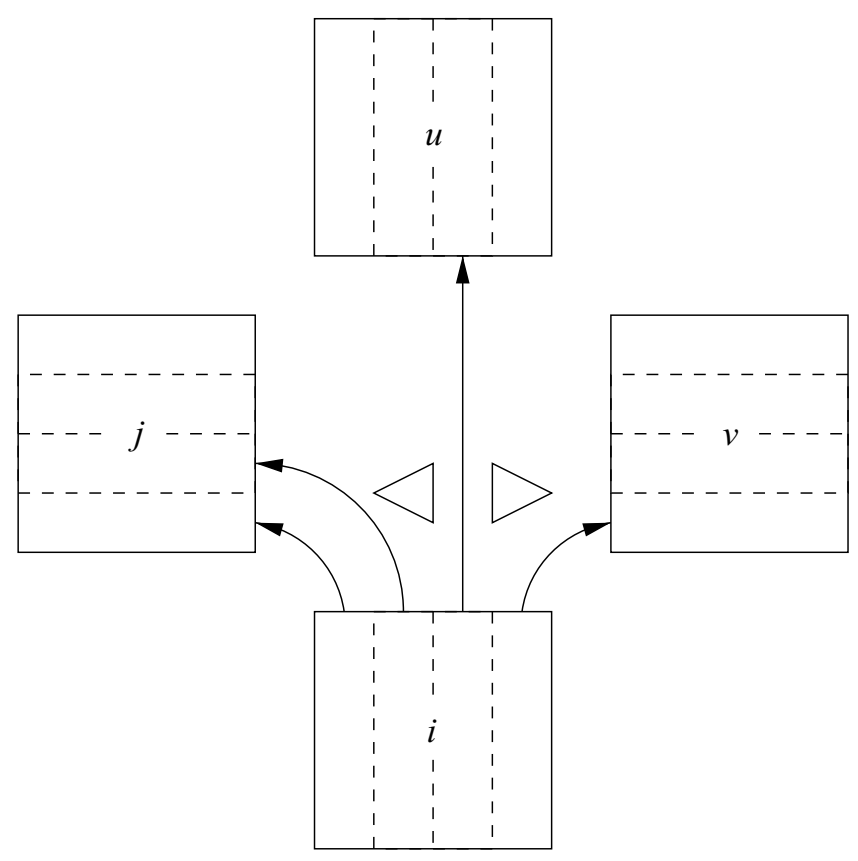

Figure 10 - Possible Routing at Intersection for $p=3$.

By limiting $p=2$ in Figure 9 evacuation organization is easier since the traffic from cell $i$ has to be splitted up only once in the middle of the street. For $p=3$ - see Figure 10 - traffic from cell $i$ has to be splitted up twice which is more difficult to organize compared to $p=2$. In addition, the network in our case study has four-way intersections at maximum so that extremely diverging traffic flows as shown in Figure 10 would result in situations where intersections are blocked for all other adjacent cells. It is important to notice that there is no "wrong" value for $p$. In fact, $p$ should be recognized as a design variable which can be chosen by the decision maker to influence the design of the evacuation plan. However, we found out that setting $p=1$ may result in some computational issues, e.g. long computation time or no feasible solution.

The procedure stops if $\left|\left(\sum_{t \in T} y_{i,|I|, t}-e_{i}\right)\right| / e_{i}<\lambda$ for all $i \in I_{S}$ or no further improvement could be achieved within a given number $(\rho)$ of succeeding iterations. We set $\lambda=0.10$ and $\rho=10$.

Due to the large network size and high problem complexity, we solved the ExCTEPM by a straight-forward two stage heuristic:

1. Step:

(a) Relax constraint (23).

(b) Remove the term $+\left(E_{i}-\sum_{\tau=1}^{t} b_{i \tau}\right)$ from (2) and (4).

(c) Reduce constraint (36) only to traffic crossing conflicts.

(d) Solve remaining problem to a maximum mipgap of $1.0 \%$ and save $\varphi_{i j}$ and $\epsilon_{i j}$ values. 
2. Step:

(a) Restore constraint (23).

(b) Restore the term $+\left(E_{i}-\sum_{\tau=1}^{t} b_{i \tau}\right)$ for (2) and (4).

(c) Solve remaining problem with $\varphi_{i j}$ and $\epsilon_{i j}$ values from step 1 as parameters.

The concept of using multiple cell sizes helps to reduce the number of cells. However, constraint (23) leads much longer computation times compared to case of using only single cell sizes. Therefore, we relax this constraint in the first step. This simplifies the network to cells with only single size. Please remember that a road network with only one cell size and $o_{j}=0.35$ would result in a network of 245 cells (236 street cells, 8 exit cells and one super sink) instead of 107 cells ( 98 street cells, 8 exit cells and one super sink) when multiple cell sizes are used. A network of 245 cells would be very time consuming to be solved compared to the network of multiple cell sizes, but only 107 cells. Of course, constraint (23) will be restored in the second step. We calculate $|T|$ analogue to the numerical example, i.e. $|T|=(8750 / 144) \cdot 1.5 \approx 91$.

The results of the second step (in terms of $\varphi_{i j}, \epsilon_{i j}$ and $\eta_{i j}$ ) are passed to the SUMO traffic simulation.

\subsection{Simulation Basics}

The ExCTEPM minimizes total danger for all evacuees during the planning horizon. In order to adopt this objective as a performance measure for the results of the microscopic traffic simulation, we used the following approach: Imagine a solution where the fraction of rescued evacuees at a particular point of time (within total evacuation time $T_{e}$ ) can be described by a curve $g(t)$ which is illustrated in Figure 11.

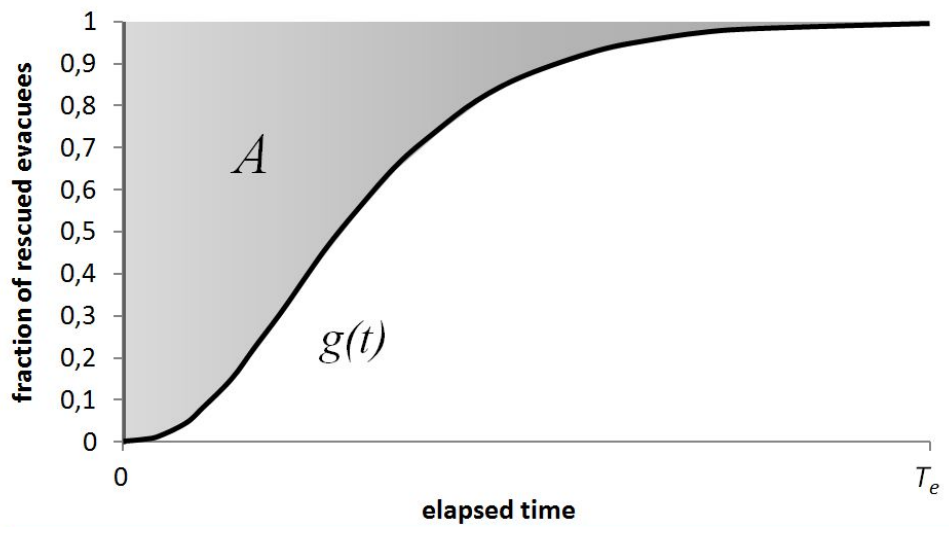

Figure 11 - Performance Measure.

The grey shaded area in Figure 11 (Area $A$ ) is an indicator of the quality of a solution, because its size depends on the number of rescued evacuees over time, described by the curve $g(t)$. 
According to the formulation of the objective function of the ExCTEPM, we additionally weight the number of rescued evacuees at a period $t$ with the amount of elapsed time. The size of this area can be calculated using equation (62):

$$
A=\int_{0}^{T_{e}}(1-g(t)) \cdot t d t
$$

We approximated (62) by the discretization

$$
A=\sum_{t=0}^{T_{e}}\left(\left(\sum_{i \in I} E_{i}\right)-g_{t}\right) \cdot t
$$

with $g_{t}$ as the number of rescued evacuees in period $t$.

In order to simplify the comparison between different solutions, we scaled all areas $A$, all total evacuation times $\left(T_{e}\right)$ and the occupation time difference between the earliest and latest exit $\left(R_{t}\right)$ by dividing them by the corresponding values of the initial solution of the simulation-based optimization procedure. As a result, area size $A$, total evacuation time $T_{e}$ as well as time range $R_{t}$ of this solution will be exactly 1 . We use SUMO 0.11.1 for all microscopic traffic simulations.

\subsection{Performance}

As a point of reference, we will use the initial solution of the procedure (Iteration 0) as a benchmark for our computational study. In particular, the occupation time difference between the earliest and latest exit $R_{t}$ (originally $R_{t}=504 \mathrm{~s}$ ), the total evacuation time $T_{e}$ (originally $T_{e}=1,346 s$ ) as well as the size of area $A$ (originally $A=1.637 \cdot 10^{9}$ ) were scaled to one. The results of the simulation-based optimization procedure are stated in Table 12 and 13:

Table 12 - Exit Occupation Times for each Iteration.

\begin{tabular}{|c|cccccccc|}
\hline Iteration & $E_{1}$ & $E_{2}$ & $E_{3}$ & $E_{4}$ & $E_{5}$ & $E_{6}$ & $E_{7}$ & $E_{8}$ \\
\hline 0 & $1,333 \mathrm{~s}$ & $1,016 \mathrm{~s}$ & $930 \mathrm{~s}$ & $913 \mathrm{~s}$ & $948 \mathrm{~s}$ & $842 \mathrm{~s}$ & $1,327 \mathrm{~s}$ & $\mathbf{1 , 3 4 6 s}$ \\
\hline 1 & $1,084 \mathrm{~s}$ & $1,084 \mathrm{~s}$ & $1,067 \mathrm{~s}$ & $1,027 \mathrm{~s}$ & $1,239 \mathrm{~s}$ & $1,276 \mathrm{~s}$ & $\mathbf{1 , 5 6 6 s}$ & $1,117 \mathrm{~s}$ \\
\hline 2 & $1,119 \mathrm{~s}$ & $1,046 \mathrm{~s}$ & $1,079 \mathrm{~s}$ & $1,090 \mathrm{~s}$ & $\mathbf{1 , 2 4 6 s}$ & $1,161 \mathrm{~s}$ & $1,148 \mathrm{~s}$ & $1,109 \mathrm{~s}$ \\
\hline 3 & $\mathbf{1 , 2 8 3 s}$ & $1,204 \mathrm{~s}$ & $1,021 \mathrm{~s}$ & $1,034 \mathrm{~s}$ & $1,057 \mathrm{~s}$ & $971 \mathrm{~s}$ & $1,132 \mathrm{~s}$ & $1,176 \mathrm{~s}$ \\
\hline 4 & $1,093 \mathrm{~s}$ & $1,011 \mathrm{~s}$ & $1,027 \mathrm{~s}$ & $1,059 \mathrm{~s}$ & $\mathbf{1 , 2 7 9 s}$ & $1,150 \mathrm{~s}$ & $1,234 \mathrm{~s}$ & $1,162 \mathrm{~s}$ \\
\hline 5 & $1,189 \mathrm{~s}$ & $1,159 \mathrm{~s}$ & $1,110 \mathrm{~s}$ & $\mathbf{1 , 1 9 0 s}$ & $1,045 \mathrm{~s}$ & $1,039 \mathrm{~s}$ & $1,109 \mathrm{~s}$ & $1,074 \mathrm{~s}$ \\
\hline
\end{tabular}

The results of the simulation-based optimization procedure are pleasing in many ways. First of all, the initial solution of the ExCTEPM (without any feedback from simulation results) shows nice results in the microscopic SUMO Traffic Simulation. Although occupation times for all exits vary quite strong, total evacuation time is relatively low. Computation time is much longer compared to succeeding iterations, because no major traffic routing decisions are preassigned in this iteration. As no major blocking of traffic streams on junctions exist, the reallocation model was applied. The evaluation of iteration 1 results in worsening of all key figures, in 
Table 13 - Performance of the Simulation-based Optimization Procedure.

\begin{tabular}{|c|ccc|c|c|}
\hline Iteration & $R_{t}$ & $T_{e}$ & $A$ & Computation Time & Event at the End of Iteration \\
\hline 0 & 1.000 & 1.000 & 1.000 & $4,318 \mathrm{~s}$ & Reallocation \\
\hline 1 & 1.069 & 1.163 & 1.137 & $454 \mathrm{~s}$ & Restore (36) \\
\hline 2 & 0.397 & 0.926 & 0.955 & $218 \mathrm{~s}$ & Reallocation \\
\hline 3 & 0.619 & 0.953 & 0.974 & $212 \mathrm{~s}$ & Reallocation \\
\hline 4 & 0.532 & 0.950 & 0.972 & $191 \mathrm{~s}$ & Reallocation \\
\hline 5 & 0.300 & 0.884 & 0.938 & $164 \mathrm{~s}$ & STOP \\
\hline
\end{tabular}

detail $R_{t}$ increases by $6.9 \%$, total evacuation time by $16.3 \%$ and the performance measure by $13.7 \%$. This deterioration is mainly caused by massive traffic blocking directly in front of a four-lane exit, leading to strong congestions during the evacuation. This observation demonstrates that traffic touching conflicts also need to be considered in evacuation planning. Due to the determination of many heavily occupied cell connections, computation time drops siginificantly compared to iteration 0 . In the following iteration, routing decisions from iteration 1 were adopted and supplemented by the re-establishment of (36) for the congested exit cell in iteration 1. This modification results in significantly better results, i.e. the best solution up to now. All key figures reach a new top mark, especially $R_{t}$ could be improved by $60.3 \%$. During the next iterations, the reallocation model was applied multiple times, finally resulting in a new best solution in iteration 5. Here, all key figures could be improved again, compared to the incumbent solution in iteration 2. $R_{t}$ reduces to $30.0 \%$ of the initial value, total evacuation time to $88.4 \%$ and the performance measure to $93.8 \%$. After iteration 5, the procedure stops since the reallocation of vehicles would result in adjustments lower than $10.0 \%$ of the original allocation. The simulation with SUMO took between 11 and 14 seconds in each iteration. A graphical representation of the number of rescueed evacuees over time can be found in Figure 12. We refused to display the charts of every iteration since the results of iteration 3 and iteration 4 are very similar to iteration 2 .

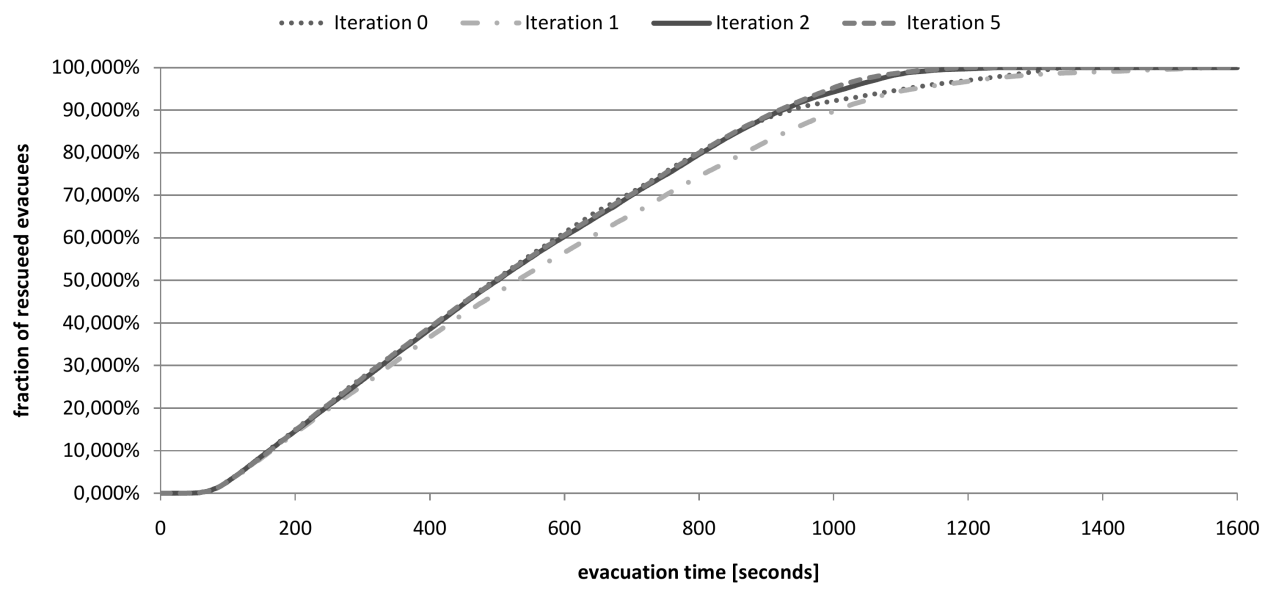

Figure 12 - Number of rescued evacuees over time. 
The initial solution of the ExCTEPM performs quite well in terms of $R_{t}, T_{e}$ and $A$. On the other hand, these results also indicate that traffic touching conflicts should be considered in the model formulation. However, the reallocation model helps to enhance the initial solution and to lower total evacuation time by more than $11 \%$.

\section{CONCLUSIONS}

Traffic routing in evacuation scenarios is a challenging problem particularly due to complex traffic dynamics. This task exacerbates, if large urban areas are the objects of research. So far, most literature only covers the evacuation of pedestrians escaping from buildings, stadiums or ships. If evacuation of urban areas is the object of interest, either relatively small areas are evacuated, low detailed network flow optimization models are applied (e.g. [28]) or simulation models (without optimization) were used.

In this paper, we develop a hybrid approach for evacuation planning on the basis of the CTEPM formulated by Kimms \& Maassen [11] and integrate three new approaches, namely multiple cell sizes, consideration of lanes as well as traffic flow limitations. The problem of determining cell sizes for real-world networks also have been discussed in this paper.

It turns out that the ExCTEPM obtains a very good initial solution in the microscopic traffic simulation. However, considering traffic touching conflicts as well as applying the vehicle reallocation model help to enhance the initial solution. Long computation times, especially in the first three iterations also reveal that there definitely exist a need for fast heuristic procedures solving the ExCTEPM.

Future research may cover the application of meta-heuristics or further experiments concerning real world applicability.

\section{ACKNOWLEDGEMENTS}

The work was done with financial support from the WestLB Stiftung Zukunft NRW. We thank AEROWEST GmbH for the written permission to use their photography of the Duisburg area in Figure 8 .

\section{REFERENCES}

[1] Andreas AK \& Smith JC. 2009. Decomposition Algorithms for the Design of a Nonsimultaneous Capacitated Evacuation Tree Network. Networks, 53: 91-103.

[2] Burstedde C, Klauck K, Schadschneider A \& Schreckenberg M. 2001. Simulation of pedestrian dynamics using a two-dimensional cellular automaton. Physica A: Statistical Mechanics and its Applications, 295: 507-525.

[3] Chakraborty J \& Armstrong MP. 1995. Using Geographic Plume Analysis to assess Community Vulnerability to Hazardous Accidents. Computers, Environment and Urban Systems, 19: 341356.

[4] Chiu Y, Zheng H, Villalobos J \& Gautam B. 2007. Modeling no-notice mass evacuation using a dynamic traffic flow optimization model. IIE Transactions, 39: 83-94. 
[5] Cova TJ \& Johnson JP. 2003. A network flow model for lane-based evacuation routing. Transportation Research Part A: General, 37: 579-604.

[6] Daganzo CF. 1994. The cell transmission model: a dynamic representation of highway traffic consistent with the hydrodynamic theory. Transportation Research Part B: Methodological, 28: 269-287.

[7] Daganzo CF. 1995. The cell transmission model, part II: network traffic. Transportation Research Part B: Methodological, 29: 79-93.

[8] Gwynne S, Galea ER, Owen M, Lawrence PJ \& Filippidis L. 1999. A review of the methodologies used in the computer simulation of evacuation from the built environment. Building and Environment, 34: 741-749.

[9] HAN LD, YUAN F, ChIN S \& HWANG H. 2006. Global optimization of emergency evacuation assignments. Interfaces, 36: 502-513.

[10] Kalafatas G \& Peeta S. 2009. Planning for Evacuation: Insights from an Efficient Network Design Model. Journal of Infrastructure Systems, 15: 21-30.

[11] Kimms A \& MaAssen K-C. 2011. Optimization and Simulation of Traffic Flows in the Case of Evacuating Urban Areas. OR Spectrum, 33: 571-593.

[12] Lee D, Kim H, PARK J-H \& PARK B-J. 2003. The current status and future issues in human evacuation from ships. Safety Science, 41: 861-876.

[13] Lighthill MJ \& Whitham JB. 1955. On kinematic waves II. A theory of traffic flow on long crowded roads. Proceedings of the Royal Society A, 229(1178): 317-345.

[14] LindeLL MK. 2008. EMBLEM2: An empirically based large scale evacuation time estimate model. Transportation Research Part A: General, 42: 140-154.

[15] LiU Y, Chang G-L, LiU Y \& Lai X. 2008. Corridor-Based Emergency Evacuation System for Washington, D.C. - System Development and Case Study. Transportation Research Record, 2041: $58-67$.

[16] Murray-Tuite PM \& Mahmassani HS. 2003. Model of Household Trip-Chain Sequencing in Emergency Evacuation. Transportation Research Record, 1831: 21-29.

[17] Murray-Tuite PM \& Mahmassani HS. 2004. Transportation Network Evacuation Planning with Household Activity Interactions. Transportation Research Record, 1894: 150-159.

[18] NAgel K \& Rickert M. 2001. Parallel implementation of the TRANSSIMS micro-simulation. Parallel Computing, 27: 1611-1639.

[19] Nagel K \& Schreckenberg M. 1992. A cellular automaton model for freeway traffic. Journal de Physique I, 2: 2221-2229.

[20] Richards PI. 1956. Shockwaves on the highway. Operations Research, 4: 42-51.

[21] Sbayti H \& Mahmassani HS. 2006. Optimal Scheduling of Evacuation Operations. Transportation Research Record, 1964: 238-246.

[22] Sheffi Y, Mahmassani H \& Powell WB. 1982. A Transportation Network Evacuation Model. Transportation Research Part A: General, 16: 209-218.

[23] Shen W, Nie Y \& Zhang HM. 2007. Dynamic Network Simplex Method for Designing Emergency Evacuation Plans. Transportation Research Record, 2022: 83-93. 
[24] SinUAny-Stern Z \& STERn E. 1993. Simulating the Evacuation of a small City: The Effects of Traffic Factors. Socio-Economic Planning Sciences, 27: 97-108.

[25] Stepanov A \& MacGregor Smith J. 2009. Multi-objective evacuation routing in transportation networks. European Journal of Operational Research, 198: 435-466.

[26] Tuydes H \& Ziliaskopoulos AK. 2006. Tabu-based heuristic approach for optimization of network evacuation contraflow. Transportation Research Record, 1964: 157-168.

[27] Xie C, Lin D-Y \& Waller T. 2010. A Dynamic Evacuation Network Optimization Problem with Lane Reversal and Crossing Elimination Strategies. Transportation Research Part E, 46: 295-316.

[28] YAMADA T. 1996. A network flow approach to a city emergency evacuation planning. International Journal of Systems Science, 27: 931-936.

[29] YAZICI MA \& Ozbay K. 2008. Evacuation Modelling in the United States: Does the Demand Model Choice Matter? Transport Reviews, 28: 757-779.

[30] Zhang J, Hodgson J \& Erkut E. 2000. Using GIS to assess the Risks of Hazardous Materials Transport in Networks. European Journal of Operational Research, 121: 316-329.

[31] Ziliaskopoulos AK. 2000. A Linear Programming Model for the Single Destination System Optimum Dynamic Traffic Assignment Problem. Transportation Science, 34: 37-49.

\section{A Postprocessing Model}

In case of non-integer vales for $\eta_{i j}$ in the optimization of the (Ex)CTEPM, this postprocessing optimization model generates a modified solution only consisting of integer values for $\eta_{i j}$. We introduce two new decision variables, namely $\eta_{i j}^{\prime}$ and $A_{i j}$. Let $\eta_{i j}^{\prime}$ be the total number of vehicles traveling from cell $i$ to cell $j$ after the postprocessing and let $A_{i j}$ be the absolute difference between the number of vehicles traveling from cell $i$ to cell $j$ before and after the postprocessing. On the basis of this notation, we can formulate the postprocessing optimization model as follows:

$$
\min \sum_{i \in I} \sum_{j \in I} A_{i j}
$$

subject to

$$
\begin{array}{ll}
\eta_{i j}-\eta_{i j}^{\prime} \leq A_{i j} & i, j \in I \\
\eta_{i j}-\eta_{i j}^{\prime} \geq-A_{i j} & i, j \in I \\
\sum_{i \in I} \eta_{i j}^{\prime}+E_{j}=\sum_{k \in I} \eta_{j k}^{\prime} & j=1, \ldots,|I|-1 \\
\sum_{i \in I} E_{i}=\sum_{i \in I} \eta_{i,|I|}^{\prime} & \\
\eta_{i j}^{\prime} \leq \eta_{i j} \cdot Y & i, j \in I \\
\eta_{i j}^{\prime} \in \mathbb{N}_{0} & i, j \in I \\
A_{i j} \geq 0 & i, j \in I
\end{array}
$$


The objective function (63) minimizes the total deviation between assigned vehicles before and after the postprocessing, whereas the deviation is calculated by constraints (64) and (65). A simple static vehicle flow equation is stated in (66) and equation (67) takes care that all vehicles will be evacuated. To ensure that no new routes will be used after the postprocessing, constraint (68) limits vehicle flows to those cell pairs where traffic occurs before the postprocessing. Here, $Y$ is a large number, e.g. $Y=\sum_{i \in I} E_{i}$. The domain of the decision variables is defined in (69) and (70).

We will demonstrate the functionality of this approach for a simple network consisting of six cells where cell 6 is the super sink, see Figure 13.

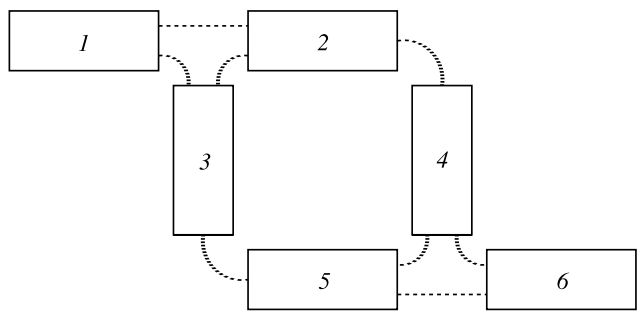

Figure 13 - Sample Network for Postprocessing.

There are 10 vehicles starting in cell 1-5 each. A possible solution with non-integer values for $\eta_{i j}$ is displayed in Figure 14. The non-integer partitioning of the vehicles leaving cell 1 affects all vehicle flows in this example, although only integer numbers of vehicles leave from cell 2-5.

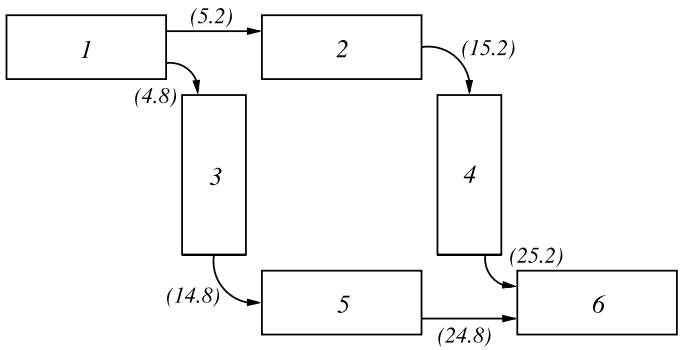

Figure 14 - Cumulated Vehicle Flows before Postprocessing.

After the postprocessing, the number of vehicles traveling between cells are only integer values, see Figure 15. The objective value for this instance is 1.2.

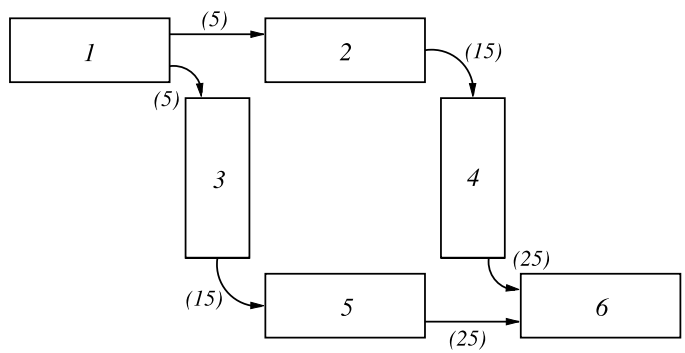

Figure 15 - Cumulated Vehicle Flows after Postprocessing. 